\title{
A Smoothness Energy without Boundary Distortion for Curved Surfaces
}

\author{
ODED STEIN, Columbia University, USA \\ ALEC JACOBSON, University of Toronto, Canada \\ MAX WARDETZKY, University of Göttingen, Germany \\ EITAN GRINSPUN, University of Toronto, Canada and Columbia University, USA
}

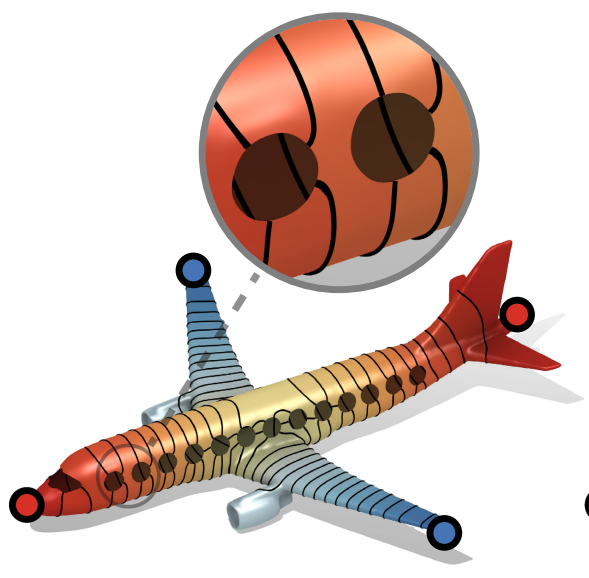

Laplacian energy

(zero Neumann boundary conditions)

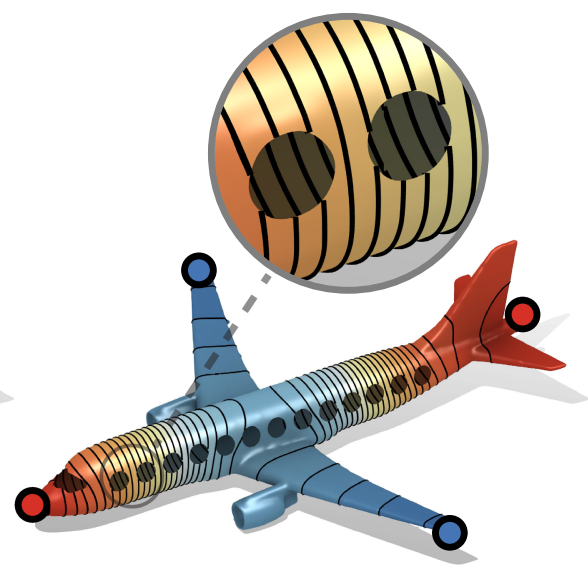

planar Hessian energy Stein et al. [2018]

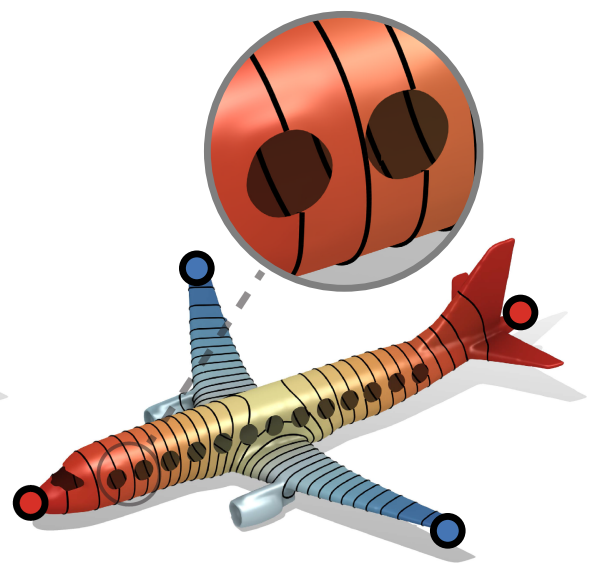

our Hessian energy

Fig. 1. Solving an interpolation problem on an airplane. Using the Laplacian energy with zero Neumann boundary conditions (left) distorts the result near the windows and the cockpit of the plane: the isolines bend so they can be perpendicular to the boundary. The planar Hessian energy of Stein et al. [2018] (center) is unaffected by the holes, but does not account for curvature correctly, leading to unnatural spacing of isolines at the front and back of the fuselage. Our Hessian energy (right) produces a natural-looking result with more regularly spread isolines, unaffected by the boundary.

Current quadratic smoothness energies for curved surfaces either exhibit distortions near the boundary due to zero Neumann boundary conditions or they do not correctly account for intrinsic curvature, which leads to unnatural-looking behavior away from the boundary. This leads to an unfortunate trade-off: One can either have natural behavior in the interior or a distortion-free result at the boundary, but not both. We introduce a

This work is funded in part by the National Science Foundation Awards CCF-17-17268 and IIS-17-17178. This research is funded in part by NSERC Discovery (RGPIN201705235, RGPAS-2017-507938), the Canada Research Chairs Program, the Fields Centre for Quantitative Analysis and Modelling and gifts by Adobe Systems, Autodesk and MESH Inc. This work is partially supported by the DFG project 282535003: Geometric curvature functionals: energy landscape and discrete methods.

Authors' addresses: O. Stein, Applied Physics and Applied Mathematics Department, 500 W. 120th St., Mudd 200, MC 4701 New York, NY 10027, USA; email: oded. stein@columbia.edu; A. Jacobson, Department of Computer Science, University of Toronto, 40 St. George Street, Rm. 4283, Toronto, Ontario, M5S 2E4, Canada; email: alecjacobson@gmail.com; M. Wardetzky, Institute of Num. and Appl. Math, University of Göttingen, Lotzestr. 16-18, 37083 Göttingen, Germany; email: wardetzky@ math.uni-goettingen.de; E. Grinspun, Department of Computer Science, University of Toronto, 40 St. George Street, Rm. 4283, Toronto, Ontario, M5S 2E4, Canada; email: eitan@cs.toronto.edu.

Permission to make digital or hard copies of all or part of this work for personal or classroom use is granted without fee provided that copies are not made or distributed for profit or commercial advantage and that copies bear this notice and the full citation on the first page. Copyrights for components of this work owned by others than the author(s) must be honored. Abstracting with credit is permitted. To copy otherwise, or republish, to post on servers or to redistribute to lists, requires prior specific permission and/or a fee. Request permissions from permissions@acm.org.

(C) 2020 Copyright held by the owner/author(s). Publication rights licensed to ACM. 0730-0301/2020/03-ART18 \$15.00

https://doi.org/10.1145/3377406 generalized Hessian energy for curved surfaces, expressed in terms of the covariant one-form Dirichlet energy, the Gaussian curvature, and the exterior derivative. Energy minimizers solve the Laplace-Beltrami biharmonic equation, correctly accounting for intrinsic curvature, leading to naturallooking isolines. On the boundary, minimizers are as-linear-as-possible, which reduces the distortion of isolines at the boundary. We discretize the covariant one-form Dirichlet energy using Crouzeix-Raviart finite elements, arriving at a discrete formulation of the Hessian energy for applications on curved surfaces. We observe convergence of the discretization in our experiments.

CCS Concepts: • Mathematics of computing $\rightarrow$ Discretization; Partial differential equations; Numerical differentiation; • Computing methodologies $\rightarrow$ Mesh geometry models;

Additional Key Words and Phrases: Geometry, biharmonic, Laplacian, Hessian, curvature, interpolation, smoothing

\section{ACM Reference format:}

Oded Stein, Alec Jacobson, Max Wardetzky, and Eitan Grinspun. 2020. A Smoothness Energy without Boundary Distortion for Curved Surfaces. ACM Trans. Graph. 39, 3, Article 18 (March 2020), 17 pages.

https://doi.org/10.1145/3377406

\section{INTRODUCTION}

Smoothness energies are used as objective functions for optimization in geometry processing. A wide variety of applications exists: 
Smoothness energies can be used to smooth data on surfaces, to denoise data, for scattered data interpolation, character animation, and much more. We are interested in quadratic smoothness energies formulated on triangle meshes.

It is desirable for a smoothing energy to have minimizers with isolines whose spacing does not vary much across the surface-the gradient of the function is sufficiently constant. When the gradient of the function is sufficiently constant, the function only changes very gradually, resulting in a smooth function. In the same vein, a good smoothing energy should have minimizers whose isolines are not distorted anywhere: Their spacing is not influenced (on the interior) by the surface's curvature, and they are not biased by the boundary of the surface-they behave locally as if the boundary were absent. Such behavior is relevant for applications where the boundary is not directly related to the actual problem that is being solved, e.g., when the boundary is an artificial result of faulty surface reconstruction resulting in a shape with many extraneous holes. One class of energies with the desired behavior in the interior are energies whose minimizers solve the biharmonic equation, the prototypical elliptic equation of order four [Gazzola et al. 2010, viii]. Such energies are pertinent as smoothness energies in computer graphics applications [Jacobson et al. 2010].

One such energy is the squared Laplacian energy-the squared Laplacian of a function integrated over the surface. We henceforth refer to the energy as simply the Laplacian energy. Its minimizers solve the biharmonic equation; as a result, they are very smooth, and their isolines behave well on curved surfaces if the surfaces are closed. The energy's most popular discretization, however, comes with zero Neumann boundary conditions. Thus, if a surface has boundaries, the minimizers are distorted near the boundary (see Figure 1), since at the boundary they are as-constant-as-possible.

The issue of boundary distortion is addressed by the Hessian energy of Stein et al. [2018]. For planar domains, they provide an energy whose minimizers solve the biharmonic equation and are aslinear-as-possible at the boundary. These boundary conditions lead to decreased distortion. The Hessian energy of Stein et al. [2018], however, is only defined for subsets of the plane $\mathbb{R}^{2}$. Stein et al. [2018] offer a way to compute an energy for curved surfaces, but, as they point out, their approach does not account for the curvature of the surface correctly. The approach of Stein et al. [2018] does not solve the biharmonic equation on curved surfaces; this leads to global distortions in the isolines of the solution (see Figure 1).

\section{Contributions}

(1) Generalized Hessian energy. We generalize the Hessian energy to accommodate curved surfaces. Our new Hessian energy is

$$
E(u):=\frac{1}{2} \int_{\Omega}(\nabla \mathrm{d} u):(\nabla \mathrm{d} u)+\kappa|\mathrm{d} u|^{2} \mathrm{~d} x,
$$

where $\nabla$ is the covariant derivative of differential forms, $\mathrm{d}$ is the exterior derivative, $\kappa$ is the Gaussian curvature, and : denotes the contraction of two operators in all indices that corresponds to $A$ : $B=\operatorname{tr}\left(A^{\top} B\right)$ (where the transpose ${ }^{\top}$ takes the metric into account). This energy

- corresponds to the Laplacian energy in the case of a domain without boundaries;
- corresponds to the Hessian energy of Stein et al. [2018] for surfaces in $\mathbb{R}^{2}, \frac{1}{2} \int_{\Omega}\left\|\overline{\mathbf{H}}_{u}\right\|_{F}^{2} \mathrm{~d} x$, where $\overline{\mathbf{H}}_{u}$ is the $2 \times 2$ Hessian matrix of $u$, and $\|A\|_{F}$ is the Frobenius norm of $A$;

- has the as-linear-as-possible natural boundary conditions of the Hessian energy of Stein et al. [2018] for flat domains in $\mathbb{R}^{2}$. These boundary conditions lead to decreased distortion at the boundary.

Figure 1 shows how our Hessian energy manages to achieve the best of both worlds.

(2) Discretization. We also introduce a discretization of this curved Hessian energy that uses Crouzeix-Raviart finite elements "under the hood," but, after the energy matrix has been assembled, relies solely on piecewise linear hat functions. We observe convergence of the discretization for a wide variety of numerical experiments, given certain regularity conditions, and apply it to various smoothing and interpolation problems.

\section{RELATED WORK}

This work extends Stein et al. [2018]. They introduce a smoothness energy with higher-order boundary conditions whose minimizers are biased less by the shape of the boundary than energies using low-order boundary conditions such as zero Neumann. Our goal is to extend their approach to curved surfaces. Section 5.3.1 mentions that their work does not correctly account for curved surfaces, and this shortcoming is addressed in this work.

\subsection{Smoothing Energies}

Smoothing energies are used for many applications in computer graphics, image processing, machine learning, and more. Quadratic smoothing energies are particularly interesting, since they are easy to work with and fast to optimize [Nocedal and Wright 2006]. The Laplacian energy is used for surface fairing and surface editing [Botsch and Kobbelt 2004; Crane et al. 2013a; Desbrun et al. 1999; Sorkine et al. 2004], for geodesic distance computation [Lipman et al. 2010], for creating weight functions used as coordinates in character animation [Jacobson et al. 2011; Weber et al. 2012], data smoothing [Weinkauf et al. 2010], image processing [Georgiev 2004], and other applications [Jacobson et al. 2010; Sýkora et al. 2014].

Geometric energies that share some of the properties of our Hessian energy have been studied in the past: In image processing, Hessian-like energies are popular for their boundary behavior, but their formulations in general do not extend to curved surfaces [Didas et al. 2009; Lefkimmiatis et al. 2011; Lysaker et al. 2003]. Similar energies are also used for data processing and machine learning but are not discretized for polyhedral meshes there [Donoho and Grimes 2003; Kim et al. 2009]. Wang et al. [2015, 2017] explicitly enforce boundary conditions on a discrete quadratic fourth-order energy to make minimizers of the energy less dependent on the boundary shape but do not discuss any continuous model corresponding to their method or which equations their minimizers satisfy.

Stein et al. [2018] present a Hessian energy for triangle meshes; however, minimizers of their discretization extended to $\mathbb{R}^{3}$ do not fulfill the biharmonic equation, leading to artifacts that are 
discussed in detail in Section 7. Liu et al. [2015] explicitly enforce higher-order boundary conditions on a smoothness energy based on a fourth-order PDE. Their energy, however, is in general not quadratic, and the boundary conditions are different than the ones presented in this article, as they are missing the as-linear-aspossible property.

A special case of a quadratic smoothness energy is the Dirichlet energy, which solves the harmonic equation $-\Delta u=0$, a simpler version of our biharmonic equation $\Delta^{2} u=0$. The Dirichlet energy can be used, for example, to create smooth character deformations [Baran and Popovic 2007; Joshi et al. 2007; Weber et al. 2007] and for image processing [Levin et al. 2004]. While the Dirichlet energy has advantages, such as a discrete maximum principle, which is preserved in some discretizations [Wardetzky et al. 2007], there are disadvantages due to the energy being first-order: Because of reduced freedom around constraints, minimizers fail to be smooth, which can lead to artifacts when applied to shape deformation [Jacobson et al. 2011, Figure 9] or worse results in image processing [Peter et al. 2016]. Higher-order smoothness energies, such as the ones derived from the biharmonic equation, are better at fitting to existing data and tend to distort results less [Georgiev 2004; Jacobson et al. 2011, 2012; Weber et al. 2012]. Additionally, the Dirichlet energy does not admit higher-order boundary conditions (unlike biharmonic energies), which makes it more difficult to use as a smoothing energy without boundary bias.

\subsection{Generalizing the Hessian Energy to Curved Surfaces}

A main theme in our work is the difficulty of generalizing expressions formulated on flat domains to curved surfaces. The presence of curvature will result in an additional term in the definition of our energy, which is absent in the planar Hessian energy of Stein et al. [2018]. This mirrors many other areas of geometry where, with the introduction of curvature, properties of flat domains cease to apply.

One such example of curvature making calculations more elaborate is parallel transport. While parallel transport of vectors is trivial on flat surfaces, this is no longer true for curved surfaces. In the presence of curvature, the parallel transport of a vector along a closed curve might result in a different vector than the initial one [Petersen 2006, pp. 156-157]. The difficulties that this phenomenon introduces to applications are discussed, for example, by Bergou et al. [2008]; Crane et al. [2010]; Polthier and Schmies [1998]; Ray et al. [2009]. Our discretization method simplifies the treatment of parallel transport by employing linear finite element basis functions that are only supported on two adjacent triangles. Since this necessitates discontinuous basis functions, this approach is less common.

Another instance of difficulties arising from the curved setting occurs in the numerical analysis of finite element methods. To apply standard finite element methods to curved surfaces, the discretization has to account for the curvature of the surface. For the case of the Poisson equation, for example, this can be either achieved by inscribing all the vertices on the limit surface while imposing triangle regularity conditions [Dziuk 1988] or by demanding a certain kind of convergence of the vertices as well as the normals of the mesh [Hildebrandt et al. 2006; Wardetzky
2006] together with specific triangle regularity conditions. Similarly, in some of our own numerical experiments, we require vertex inscription and the triangle regularity condition to achieve convergence.

\subsection{Discretization of the Vector Dirichlet Energy}

An important part of the discretization of our curved Hessian energy is the discretization of the vector Dirichlet energy $\frac{1}{2} \int_{\Omega} \nabla \mathbf{v}$ : $\nabla \mathbf{v} \mathrm{d} x$, where $\nabla$ is the covariant derivative. The problem of discretizing the covariant derivative for surfaces in general, and the vector Dirichlet energy on surfaces in particular, are active areas of research. Knöppel et al. [2013] provide a finite element discretization of the vector Dirichlet energy that places the degrees of freedom on mesh vertices. This discretization is used to design direction fields. A different discretization, reminiscent of finite differences, can be found in the work of Knöppel et al. [2015], where it is used to compute stripe patterns on surfaces. The same discretization is also used by Sharp et al. [2018] to compute the parallel transport of vectors. The work of Sharp et al. [2018] also features the Weitzenböck identity that we use to derive the natural boundary conditions of our Hessian energy: They use it to construct a Dirichlet energy on the covector bundle. Liu et al. [2016] discretize the covariant derivative using the notion of discrete connections. They use it to improve the quality of the vector fields produced by Knöppel et al. [2013] and provide some evidence of convergence. Other examples of discretizations of the covariant derivative include Azencot et al. [2015], who compute the directional derivatives of each of the vector field's component functions, and Corman and Ovsjanikov [2019], who leverage a functional representation to compute covariant derivatives.

To simplify computation, we propose an alternative discretization of the vector Dirichlet energy. We use the scalar CrouzeixRaviart finite element, the "simplest nonconforming element for the discretization of second order elliptic boundary-value problems" [Braess 2007, p. 109]. It was first introduced by Crouzeix and Raviart [1973] and has become a very popular finite element for the nonconforming discontinuous Galerkin method. It is known to converge for the scalar Poisson equation in $\mathbb{R}^{2}$. Unlike the discretizations mentioned above, the degrees of freedom are placed on the mesh edges. The Crouzeix-Raviart finite element has been popular in computer graphics applications such as the works of Bergou et al. [2006]; Brandt et al. [2018]; English and Bridson [2008]; Vaxman et al. [2016, Section 4.2].

Crouzeix-Raviart elements are simpler than the finite elements of Knöppel et al. [2013], but they come at a cost: The basis functions are discontinuous, and the method cannot be used for applications where the vectors have to live on vertices. In our application, the vector-valued functions are only intermediates, so we have more freedom in choosing their discretization and to put vectors on edges.

The discretization of one-forms using the Crouzeix-Raviart finite element presented in this work is closely related to other generalizations of the Crouzeix-Raviart element to vector- and differential-form-like quantities such as those present in the work of Wardetzky [2006] and those discussed in the survey of Brenner [2015]. 


\section{SMOOTHNESS ENERGIES}

A classical smoothness energy for a surface $\Omega \subseteq \mathbb{R}^{3}$ is the Laplacian energy with zero Neumann boundary conditions. When using this method, one solves the optimization problem

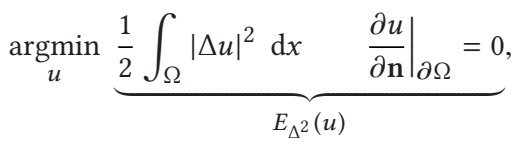

where $\Delta$ is the Laplace-Beltrami operator, and $\left.\frac{\partial u}{\partial n}\right|_{\partial \Omega}$ is the normal derivative at the boundary. $\left.\frac{\partial u}{\partial \mathrm{n}}\right|_{\partial \Omega}=0$ is the zero Neumann boundary condition. In practice, when minimizing this energy by directly discretizing it and then optimizing the resulting quadratic form, the boundary conditions manifest as an implicit penalty on the gradient of the function at the boundary during optimization. We will refer to the whole optimization problem with zero Neumann boundary conditions by $E_{\Delta^{2}}$. Minimizers of the Laplacian energy solve the biharmonic equation $\Delta^{2} u=0$. This leads to natural-looking, smooth results on the interior. ${ }^{1}$ The energy is easy to discretize even for meshes that are non-planar using methods such as the mixed finite element method (FEM) [Jacobson et al. 2010]. Using this method, the zero Neumann boundary condition does not need to be imposed on top of the discretization; it is simply "baked in" by squaring the classical cotan Laplacian. The cotan Laplacian is also known as the Lagrangian linear FEM for the Poisson equation (it goes back to Duffin [1959] and MacNeal [1949], and its convergence for the Poisson equation was shown by Dziuk [1988]).

The minimizers of $E_{\Delta^{2}}$, however, are biased by the shape of the boundary. Their isolines are significantly distorted near the domain boundary: They are perpendicular to it, as they have to fulfill the zero Neumann boundary conditions (as-constant-aspossible). Simply removing the zero Neumann boundary conditions and minimizing the Laplacian energy without any boundary conditions is not a good alternative. Minimizations without explicit boundary conditions lead to natural boundary conditions. The natural boundary conditions of the Laplacian energy are too permissive [Stein et al. 2018, Figure 3]. This behavior is one of the motivations for the Hessian energy of Stein et al. [2018]. It is formulated as the following minimization problem. For a surface $U \subseteq \mathbb{R}^{2}$,

$$
\underset{u}{\operatorname{argmin}} \underbrace{\frac{1}{2} \int_{U} \overline{\mathbf{H}}_{u}: \overline{\mathbf{H}}_{u} \mathrm{~d} x}_{E_{\overline{\mathrm{H}}^{2}}(u)},
$$

where $\overline{\mathbf{H}}_{u}$ is the $2 \times 2$ Hessian matrix of $u$, and $\mathbf{A}: \mathbf{B}=\operatorname{tr}\left(\mathbf{A}^{\top} \mathbf{B}\right)$. Minimizers of this energy solve the biharmonic equation in $\mathbb{R}^{2}$. Its natural boundary conditions lead to as-linear-as-possible behavior on the boundary. This makes minimizers less biased than the zero Neumann boundary condition.

Stein et al. [2018] demonstrate the benefits of the natural boundary conditions of the Hessian energy with applications for curved

${ }^{1}$ Of course, simply minimizing Equation (2) results in the zero function. However when combined with additional Dirichlet boundary conditions, this gives a nontrivial result for the biharmonic equation $\Delta^{2} u=0$, and, when combined with the additional energy term $\int_{\Omega} u f \mathrm{~d} x$ it gives a result for the biharmonic Poisson-type equation $\Delta^{2} u=f$

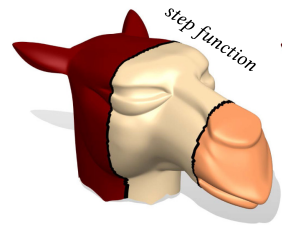

input

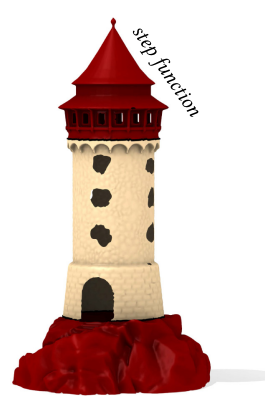

Fig. 2. Smoothing a step function (left) on a surface using the method of Stein et al. [2018] (middle) does not correctly account for the curvature of the surface, leading to crooked isolines. Our curved Hessian energy $E$ (right) correctly accounts for curvature and does not suffer from such problems.

surfaces in $\mathbb{R}^{3}$ as well. Their discretization of the planar Hessian energy for curved surfaces is achieved by extending every operator involved in the $\mathbb{R}^{2}$ discretization to three dimensions. This approach (the discretization, as well as the smooth formulation) does not account for the curvature of surfaces correctly, and its minimizers do not solve the biharmonic equation on curved surfaces [Stein et al. 2018, Section 5.3.1]. We refer to this generalization as the planar Hessian energy $E_{\overline{\mathbf{H}}^{2}}$ when talking about it in the context of curved surfaces. This planar Hessian energy is suitable for some applications but leads to global deviations from the naturallooking isolines produced by $E_{\Delta^{2}}(u)$ (see Figure 1) or an implementation of the Hessian energy that does account for curvature (see Figure 2) in others.

\section{WARM-UP: THE DIRICHLET ENERGY ON CURVED SURFACES}

As a warm-up, we consider the simple and well-known Dirichlet energy: It is easy to generalize to curved surfaces. We will perform the calculation for this generalization here. The calculation is wellknown, and this didactic exercise will inform our generalization of the planar Hessian energy to curved surfaces later.

\subsection{From the Energy to the PDE}

Let $H^{k}$ denote the Sobolev space of real-valued functions with $k$ weak derivatives in $L^{2}$. The Dirichlet energy for domains $U \subseteq \mathbb{R}^{2}$ is defined, for $u \in H^{2}(U)$, as ${ }^{2}$

$$
E_{\bar{\nabla}^{2}}(u):=\frac{1}{2} \int_{\Omega} \bar{\nabla} u \cdot \bar{\nabla} u \mathrm{~d} x,
$$

\footnotetext{
${ }^{2}$ We choose to formulate this energy for $u \in H^{2}(U)$, although it is well-defined for $u \in H^{1}(U)$, since we will continue our calculations with the same $u$ right away, and we will need additional smoothness.
} 
where $\bar{\nabla}$ is the vector of partial derivatives of $u, \bar{\nabla} u=$ $\left(\begin{array}{ll}\partial_{x} u & \partial_{y} u\end{array}\right)^{\top}$, the normal two-dimensional gradient in $\mathbb{R}^{2}$.

Minimizers of the Dirichlet energy solve the Laplace equation [Evans 2010]. Indeed, consider the variation

$$
u \rightarrow u+h v \quad u, v \in H^{2}(U)
$$

for some $h>0$. Since our functions are in the Sobolev function space $H^{2}$, we can differentiate them at least twice. Plugging the variation into $E_{\bar{\nabla}^{2}}(u)$, differentiating with respect to $h$, and then setting $h=0$, we can see that a minimizer $u$ must fulfill the equation

$$
\int_{U}\left(\partial_{i} u\right)\left(\partial_{i} v\right) \mathrm{d} x=0 \quad \forall v \in H^{2}(U),
$$

where $\partial_{*}$ is a partial derivative, and summation over repeated indices is implied. This is a standard technique of variational calculus. Using integration by parts (where $\mathbf{n}$ is the boundary normal)

$$
\begin{aligned}
0 & =\int_{U}\left(\partial_{i} u\right)\left(\partial_{i} v\right) \mathrm{d} x \\
& =\int_{\partial U}\left(\partial_{i} u\right) v \mathbf{n}_{i} \mathrm{~d} x-\int_{U}\left(\partial_{i} \partial_{i} u\right) v \mathrm{~d} x .
\end{aligned}
$$

Here, a boundary term appeared as a result of integration by parts. The second term of the second line corresponds to the standard two-dimensional planar Laplacian $\bar{\Delta}=\bar{\nabla} \cdot \bar{\nabla}$, and so we conclude that minimizers of the energy $E_{\bar{\nabla}^{2}}(u)$ fulfill the two-dimensional planar Laplace equation $-\bar{\Delta} u=0$. The additional boundary term, the first term of the second line in Equation (6), determines the natural boundary conditions of the Dirichlet energy. They are called natural boundary conditions, because they naturally emerge from solving the variational problem over the set of all functions without explicitly enforcing additional boundary conditions. In this case, we can see that the natural boundary conditions are zeroNeumann boundary conditions:

$$
\partial_{i} u \mathbf{n}_{i}=\bar{\nabla} u \cdot \mathbf{n}=0 \quad \text { on } \partial U .
$$

\subsection{From the PDE to a New Energy}

We now generalize the Dirichlet energy to curved surfaces. This means we are looking for an energy whose minimizers solve a curved version of the Laplace equation and fulfill a curved version of the natural boundary conditions (7). While we were able to write the calculations in terms of coordinates in the flat setting, this is much harder to do in the curved setting. This is why we perform calculations in the curved setting in a coordinate-free fashion.

The curved analog of the planar Laplace equation is $\Delta u=0$, where $\Delta$ is the Laplace-Beltrami operator [Jost 2011, Chapter 3]. It holds for a function $u \in H^{2}(\Omega)$ (where $\Omega$ is a compact surface immersed in $\mathbb{R}^{3}$ ) that

$$
\Delta u=\delta \mathrm{d} u,
$$

where $\mathrm{d}$ is the exterior derivative and $\delta$ is the codifferential, the (formal) dual of the exterior derivative under integration by parts. For planar surfaces, the Laplace-Beltrami operator $\Delta$ corresponds to $-\bar{\Delta}$.

We start with an integral formulation of the Laplace equation and then use integration by parts. For all $v \in H^{2}(\Omega)$ it must hold that

$$
\begin{aligned}
0 & =\int_{\Omega}(\Delta u) v \mathrm{~d} x=\int_{\Omega}(\delta \mathrm{d} u) v \mathrm{~d} x \\
& =-\int_{\partial \Omega}\langle\mathrm{d} u, \mathbf{n}\rangle v \mathrm{~d} x+\int_{\Omega}(\mathrm{d} u) \cdot(\mathrm{d} v) \mathrm{d} x,
\end{aligned}
$$

where the natural (metric-independent) pairing of one-forms and vectors is indicated using the angle bracket, and · is the dot product of one-forms.

Using the definition of the gradient $\nabla$ on curved surfaces, $\nabla u \cdot \mathbf{w}:=\langle\mathrm{d} u, \mathbf{w}\rangle$ for a vector $\mathbf{w}$ (where $\cdot$ is the dot product of vectors and the angle bracket $\langle\cdot, \cdot\rangle$ denotes the pairing of a one-form with a vector) [Jost 2011, (3.1.16)], we can write

$$
0=-\int_{\partial \Omega} \nabla u \cdot \mathbf{n} v \mathrm{~d} x+\int_{\Omega} \nabla u \cdot \nabla v \mathrm{~d} x
$$

Walking back through the variation from Equation (5), this now motivates the definition of a curved Dirichlet energy

$$
E_{\nabla^{2}}(u):=\frac{1}{2} \int_{\Omega} \nabla u \cdot \nabla u \mathrm{~d} x .
$$

We have shown that minimizers of this energy solve the curved Laplace equation, and by the boundary term in Equation (9) it is also clear that minimizers fulfill a curved zero Neumann boundary condition:

$$
\nabla u \cdot \mathbf{n}=0 \quad \text { on } \partial U
$$

Thus, we have successfully generalized the Dirichlet energy to curved surfaces. Even though we went through the work of using differential geometric operators, we ended up with something quite similar to what we started with, but with $\bar{\nabla}$ replaced by $\nabla$. For more complicated energies this will no longer be the case.

\section{THE HESSIAN ENERGY ON CURVED SURFACES}

We now seek to derive a smooth Hessian energy on surfaces that generalize the Hessian energy in $\mathbb{R}^{2}$ while ensuring that minimizers of the energy solve the biharmonic equation. This will follow the approach we used in Section 4 to generalize the planar Dirichlet energy to curved surfaces.

\subsection{From the Energy to the PDE}

For the planar Hessian energy $E_{\overline{\mathrm{H}}^{2}}$ it is a straightforward calculation to prove that minimizers fulfill the biharmonic equation. This calculation is mentioned, for example, in Stein et al. [2018, Section 4], and we will repeat it here for convenience. Our setting is a compact planar domain $U \subseteq \mathbb{R}^{2}$. The linear equation fulfilled by minimizers of Equation (3) derived with standard variational calculus is: find $u \in H^{4}(U)$ such that

$$
\int_{U}\left(\partial_{i} \partial_{j} u\right)\left(\partial_{i} \partial_{j} v\right) \mathrm{d} x=0 \quad \forall v \in H^{4}(U)
$$

where, as before, $\partial_{*}$ is a partial derivative, and summation over repeated indices is implied. Using integration by parts (where $\mathbf{n}$ is 
the boundary normal), we know that

$$
\begin{aligned}
0= & \int_{U}\left(\partial_{i} \partial_{j} u\right)\left(\partial_{i} \partial_{j} v\right) \mathrm{d} x \\
= & \int_{\partial U}\left(\partial_{i} \partial_{j} u\right)\left(\partial_{j} v\right) \mathbf{n}_{i} \mathrm{~d} x-\int_{U}\left(\partial_{i} \partial_{i} \partial_{j} u\right)\left(\partial_{j} v\right) \mathrm{d} x \\
= & \int_{\partial U}\left(\partial_{i} \partial_{j} u\right)\left(\partial_{j} v\right) \mathbf{n}_{i}-\left(\partial_{i} \partial_{i} \partial_{j} u\right) v \mathbf{n}_{j} \mathrm{~d} x \\
& +\int_{U}\left(\partial_{j} \partial_{i} \partial_{i} \partial_{j} u\right) v \mathrm{~d} x
\end{aligned}
$$

Since all partial derivatives commute in the plane, in the very last term, we can write $\partial_{j} \partial_{i} \partial_{i} \partial_{j} u=\partial_{i} \partial_{i} \partial_{j} \partial_{j} u=\bar{\Delta}^{2} u$. As a result, we can conclude that minimizers of the Hessian energy satisfy the biharmonic equation with some additional boundary terms. This commutation will not be that easy for curved surfaces.

After some rearranging, these boundary terms can be seen to imply the natural boundary conditions

$$
\begin{aligned}
\mathbf{n}^{\top} \overline{\mathbf{H}}_{u} \mathbf{n}=0 & \text { on } \partial U \\
\bar{\nabla} \Delta u \cdot \mathbf{n}+\bar{\nabla}\left(\mathbf{t}^{\top} \overline{\mathbf{H}}_{u} \mathbf{n}\right) \cdot \mathbf{t}=0 & \text { on } \partial U,
\end{aligned}
$$

where $\mathbf{n}$ is the normal vector at the boundary, and $\mathbf{t}$ is the tangential vector of the (oriented) boundary. A derivation of Equation (14) can be found in the work of Stein et al. [2018, Section 4.3].

A naive approach to a Hessian energy for curved surfaces. Since our goal is to generalize the Hessian energy for surfaces, it seems natural to simply replace the planar Hessian $\overline{\mathbf{H}}_{u}$ with an analog for curved surfaces and minimize this generalization of the Hessian energy. Unfortunately, this will not work: The resulting minimizers of such an energy will not solve the biharmonic equation.

Consider a compact surface $\Omega$ immersed in $\mathbb{R}^{3}$. We define the Hessian of a function on a curved surface [Lee 1997, p. 54]

$$
\mathrm{H}_{u}:=\nabla \mathrm{d} u,
$$

where $\nabla$ applied to one-forms is the covariant derivative of differential forms and $d$ is the exterior derivative. It might seem reasonable to define a generalized Hessian energy as

$$
E_{\mathbf{H}^{2}}(u):=\frac{1}{2} \int_{\Omega} \mathbf{H}_{u}: \mathbf{H}_{u} \mathrm{~d} x,
$$

where : now denotes the contraction of all indices. The associated variational equation at a stationary point is

$$
\int_{\Omega}(\nabla \mathrm{d} u):(\nabla \mathrm{d} v) \mathrm{d} x=0 \quad \forall v \in H^{4}(\Omega) .
$$

We can already see that we will not be able to repeat our approach from Equation (13): There is no way to easily commute $\nabla$ and $d$, as it was possible in the flat setting with coordinate-wise calculation, and thus, we ca not perform the same simple calculation to show that minimizers of $E_{\mathrm{H}^{2}}$ solve the biharmonic equation.

\subsection{From the PDE to a New Energy}

Instead, echoing Section 4.2, we derive an energy whose minimizers fulfill the boundary conditions (14) and also solve the biharmonic equation. We start with the integrated biharmonic equation using the Hodge Laplacian operator $\Delta=\mathrm{d} \delta+\delta \mathrm{d}$ for forms on surfaces, which degenerates to the Laplace-Beltrami operator $\delta \mathrm{d}$ for

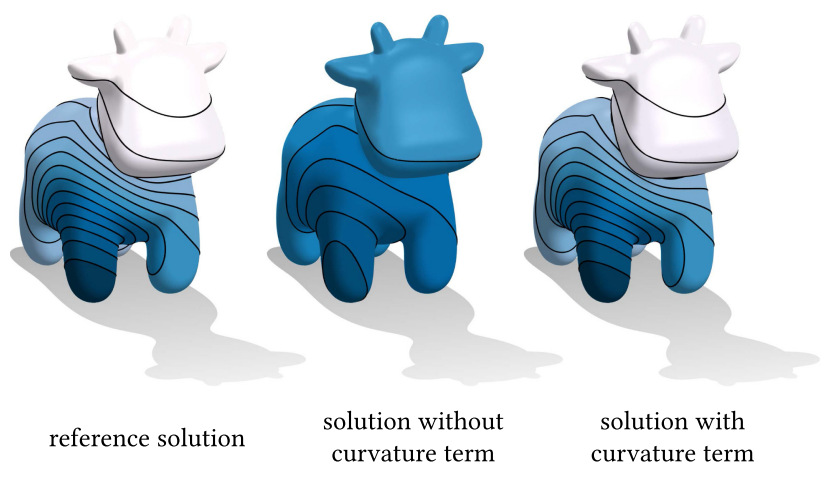

Fig. 3. We solve the Poisson-like problem $\Delta^{2} u=f$ using the Hessian energy with (right, E) and without (center, $E_{\mathrm{H}^{2}}$ ) curvature term. The solution for $E_{\Delta^{2}}$ is provided as a reference solution (left). We see that the solution for $E$ corresponds to the reference solution, since its minimizers solve the biharmonic equation, while the solution for $E_{\mathrm{H}^{2}}$ does not.

zero-forms (scalar functions) and which corresponds to the standard Laplacian for functions in the plane. It holds that

$$
\begin{aligned}
0 & =\int_{\Omega}(\Delta \Delta u) v \mathrm{~d} x=\int_{\Omega}(\delta \mathrm{d} \delta \mathrm{d} u) v \mathrm{~d} x \\
& =-\int_{\partial \Omega}\langle\mathrm{d} \delta \mathrm{d} u, \mathbf{n}\rangle v \mathrm{~d} x+\int_{\Omega}(\mathrm{d} \delta \mathrm{d} u) \cdot(\mathrm{d} v) \mathrm{d} x,
\end{aligned}
$$

where $\mathbf{n}$ is the boundary normal vector, and we used the fact that the exterior derivative $\mathrm{d}$ is dual to the codifferential $\delta$.

Now, we utilize the Weitzenböck identity. It relates the HodgeLaplacian $\Delta=\mathrm{d} \delta+\delta \mathrm{d}$ and the Bochner Laplacian $\Delta_{B}=\nabla^{*} \nabla$, where $\nabla^{*}$ is the (formal) dual covariant derivative. The formal dual is defined via integration by parts on a closed manifold $M$, $\int_{M} X: \nabla \omega \mathrm{d} x=\int_{M} \nabla^{*} X \cdot \omega \mathrm{d} x$. It holds that

$$
\Delta=\nabla^{*} \nabla+\text { Ric }
$$

where Ric is the Ricci curvature tensor [Petersen 2006, Chapter 7]. This formula dates back to Bochner [1946] and Weitzenböck [1885]. It is used, together with the fact that $\mathrm{d}^{2}=0$, to continue our calculation from Equation (17).

$$
\begin{aligned}
\int_{\Omega}(\mathrm{d} \delta \mathrm{d} u) \cdot(\mathrm{d} v) \mathrm{d} x= & \int_{\Omega}((\mathrm{d} \delta+\delta \mathrm{d}) \mathrm{d} u) \cdot(\mathrm{d} v) \mathrm{d} x \\
= & \int_{\Omega}\left(\nabla^{*} \nabla \mathrm{d} u\right) \cdot(\mathrm{d} v)+\operatorname{Ric}(\mathrm{d} u, \mathrm{~d} v) \mathrm{d} x \\
= & -\int_{\partial \Omega} \mathrm{n}^{i}(\nabla \mathrm{d} u)_{i j} \cdot(\mathrm{d} v)_{j} \mathrm{~d} x \\
& +\int_{\Omega}(\nabla \mathrm{d} u):(\nabla \mathrm{d} v)+\operatorname{Ric}(\mathrm{d} u, \mathrm{~d} v) \mathrm{d} x
\end{aligned}
$$

where indices have been added to make clear which contraction happens in which index.

The term involving the Ricci curvature tensor Ric can be further simplified. For the case of two-dimensional manifolds, we know that we can write the Ricci curvature tensor as simply

$$
\text { Ric }=\kappa g,
$$

where $\kappa$ is the Gaussian curvature, i.e., half the scalar curvature [Petersen 2006, pp. 38-41]. 
Putting Equations (17), (19), and (20) together then gives

$$
\begin{aligned}
0=- & \int_{\partial \Omega}\langle\mathrm{d} \delta \mathrm{d} u, \mathbf{n}\rangle v+\mathbf{n}^{i}(\nabla \mathrm{d} u)_{i j} \cdot(\mathrm{d} v)_{j} \mathrm{~d} x \\
& +\int_{\Omega}(\nabla \mathrm{d} u):(\nabla \mathrm{d} v)+\kappa \mathrm{d} u \cdot \mathrm{d} v \mathrm{~d} x .
\end{aligned}
$$

This is, in the case of a planar surface (for which it holds $\kappa=0$ ), exactly the term from our earlier calculation with the planar Hessian energy from Equation (13). Here, we also see why minimizers of the naive Hessian energy $E_{\mathrm{H}^{2}}$ do not solve the biharmonic equation on curved surfaces: The energy $E_{\mathbf{H}^{2}}$ lacks the curvature correction term involving $\kappa$ (see Figure 3).

The result from Equation (21) motivates the definition of the following curved Hessian energy:

$$
E(u):=\frac{1}{2} \int_{\Omega}(\nabla \mathrm{d} u):(\nabla \mathrm{d} u)+\kappa|\mathrm{d} u|^{2} \mathrm{~d} x .
$$

Minimizers of the energy $E$ solve the biharmonic equation on a surface in $\mathbb{R}^{3}$, unlike minimizers of $E_{\mathrm{H}^{2}}$.

It remains to check what the natural boundary conditions of $E$ are. We can find them by checking which biharmonic functions $u$ fulfill the boundary terms

$$
0=\int_{\partial \Omega}\langle\mathrm{d} \delta \mathrm{d} u, \mathbf{n}\rangle v+\mathbf{n}^{i}(\nabla \mathrm{d} u)_{i j} \cdot(\mathrm{d} v)_{j} \mathrm{~d} x \quad \forall v \in H^{4}(\Omega) .
$$

We use the same strategy as Stein et al. [2018, Section 4.3]: testing with specific subsets of all valid test functions. These subsets are purpose-built to expose the natural boundary conditions of the energy. First, we test with all functions $v$ that vanish on the boundary, and thus only have nonzero differential in the normal direction $(v=0,\langle\mathrm{~d} v, \mathbf{w}\rangle=g \mathbf{n} \cdot \mathbf{w}$ for some smooth $g)$. It follows that

$$
\mathbf{n}^{i}(\nabla \mathrm{d} u)_{i j} \mathbf{n}^{j}=0 \quad \text { on } \partial \Omega,
$$

i.e., the (curved) Hessian of the solution is linear across the boundary; the second derivative of the function across the boundary is zero. This mirrors the "as-linear-as-possible" condition of Stein et al. [2018, (17)].

Using the same strategy of testing the expression with a specific subset of functions to expose boundary behavior, if we plug in all functions that have zero differential in the normal direction at the boundary $(\langle\mathrm{d} v, \mathbf{n}\rangle=0)$, we get

$$
\langle\mathrm{d} \delta \mathrm{d} u, \mathbf{n}\rangle+\delta_{\partial \Omega, j} l \partial \Omega\left(\mathbf{n}^{i}(\nabla \mathrm{d} u)_{i j}\right)=0 \quad \text { on } \partial \Omega,
$$

where $l_{\partial \Omega}$ is the natural projection of one-forms on the surface to one forms on the boundary, and the subscript on the codifferential implies that this is the codifferential of the boundary manifold in the index $j$. This mirrors the condition from Stein et al. [2018, (18)]. In fact, the two natural boundary conditions (23) and (24) of the Hessian energy are exactly the ones of the planar Hessian energy if the domain is a planar surface.

The Hessian energy natural boundary conditions. Like the natural boundary conditions of $E_{\overline{\mathbf{H}}^{2}}$ from Stein et al. [2018, Section 4.3], the natural boundary conditions (23) and (24) of the Hessian energy $E$ guarantee that its minimizers

- continue linearly across the boundary in the normal direction $\left(\mathbf{n}^{i}(\nabla \mathrm{d} u)_{i j} \mathbf{n}^{j}=0\right)$, and

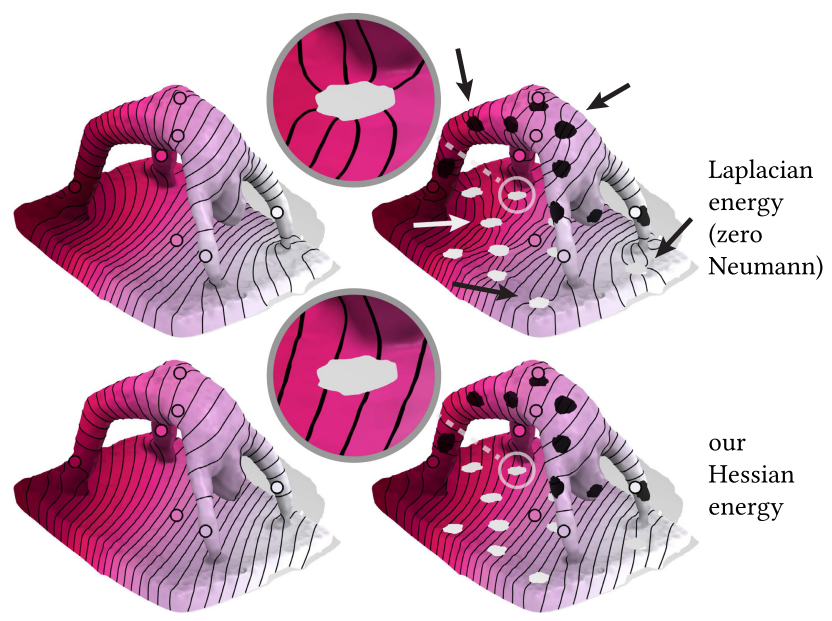

Fig. 4. Using the Laplacian energy $E_{\Delta^{2}}$ (top) for scattered data interpolation gives a result that is influenced by the boundary: Adding holes makes the isolines near them bend towards the holes. Our Hessian energy $E$ (bottom) is less distorted at the holes and produces a very similar result without and with holes.

- have limited variation along the boundary $\left(\langle\mathrm{d} \delta \mathrm{d} u, \mathbf{n}\rangle+\delta_{\partial \Omega, j} l \partial \Omega\left(\mathbf{n}^{i}(\nabla \mathrm{d} u)_{i j}\right)=0\right)$,

as discussed by Stein et al. [2018, Section 4.3]. Both boundary conditions are fulfilled by minimizers of $E$ in the absence of explicitly enforced boundary conditions.

On planar surfaces, these boundary conditions mean that the null space of the energy contains all linear functions, in contrast to the Laplacian energy with zero Neumann boundary conditions $E_{\Delta^{2}}$, whose null space only contains constant functions. On closed surfaces, the null space of $E$ and $E_{\Delta^{2}}$ is the same: all constant functions.

The natural boundary conditions of the Hessian energy have a physical interpretation. Consider a deforming flat thin plate where displacement is modeled by the function $u$. The plate is not clamped or supported at the boundary in any way: It is a free plate. Then the conditions (24) are the boundary conditions fulfilled by $u$ [Courant and Hilbert 1924, pp. 206-207]. These boundary conditions go back at least as far as Rayleigh [1894, p. 355].

Its natural boundary conditions make the Hessian energy a good choice for ignoring the boundary as much as possible while maintaining biharmonic behavior everywhere away from the boundary (see Figure 4, where they are contrasted with zero Neumann boundary conditions).

\section{DISCRETIZATION}

We offer a discretization for the curved Hessian energy $E$ derived in Section 5. The approach presented here is a simple method using only linear finite elements, intended to make the Hessian energy easily accessible. There are, however, other conceivable ways to discretize this energy, such as, for example, higher-order conforming finite elements [Braess 2007, II.5].

\subsection{Computing the Hessian Energy}

Discretizing the Hessian energy $E$ (22) as written would require us to discretize functions that can be differentiated twice. To avoid 


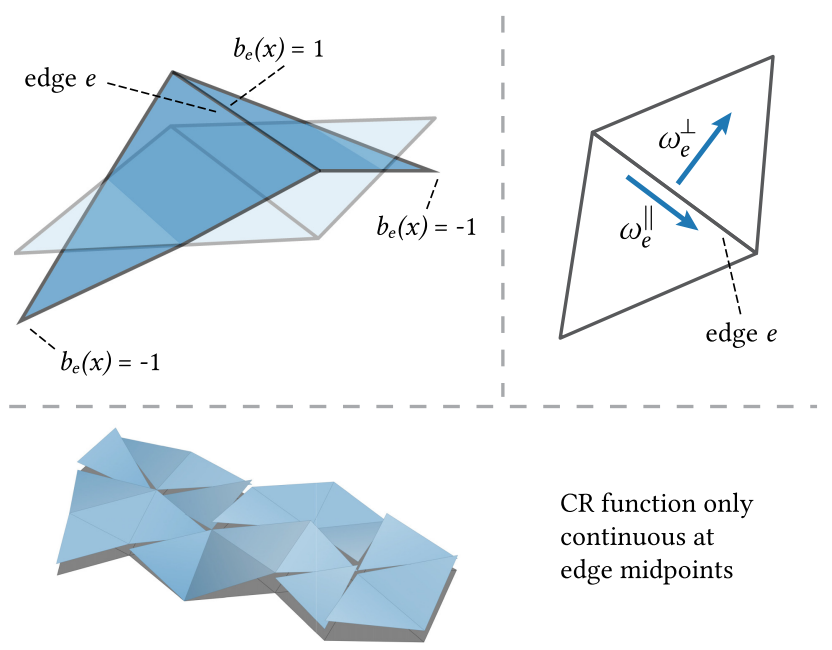

Fig. 5. A scalar Crouzeix-Raviart basis function for the edge $e$ (top left). The parallel and perpendicular one-forms for the edge $e$, represented by their dual vectors (top right). Crouzeix-Raviart functions and their sums are, in general, discontinuous. Continuity is only guaranteed at edge midpoints (bottom).

this complication, we use the mixed finite element method [Boffi et al. 2013] by introducing an intermediate variable $w=\mathrm{d} u$ and formulate the problem of minimizing $E$ as

$$
\underset{u}{\operatorname{argmin}} \frac{1}{2} \int_{\Omega}(\nabla w):(\nabla w)+\kappa|w|^{2} \mathrm{~d} x, \quad w=\mathrm{d} u .
$$

Using Lagrange multipliers to enforce the constraint $w=\mathrm{d} u$, we can write the optimization problem as the saddle problem (where our goal is finding a stationary point)

$$
\begin{gathered}
\underset{\substack{\text { saddle } \\
u, \lambda}}{\frac{1}{2}} \int_{\Omega}(\nabla w):(\nabla w)+\kappa|w|^{2} \mathrm{~d} x \\
-\int_{\Omega} \lambda \cdot(w-\mathrm{d} u) \mathrm{d} x .
\end{gathered}
$$

We discretize the space of scalar functions (containing $u$ ) using standard continuous, piecewise linear functions, which are a very commonly used finite element. Definitions are found, for example, in Braess [2007, II.5]. The basis of this discrete space consists of the $\varphi_{i}, i=1, \ldots n$, sometimes called "hat functions" (see inset).

We write $u=\sum_{i} u_{i} \varphi_{i}$, and we have the vector $\mathbf{u}=$ $\left(u_{1}, \ldots, u_{n}\right)^{\top}$.

The space of one-forms (containing $w)$ is discretized using Crouzeix-Raviart one-forms (CROFs), which are described in Section 6.2. The basis of this discrete space are the functions $\eta_{i}, i=1, \ldots m$. We write $w=\sum_{i} w_{i} \eta_{i}$, and we have the

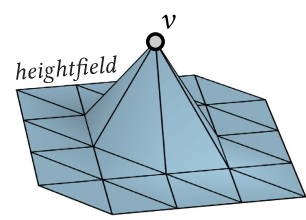
vector $\mathbf{w}=\left(w_{1}, \ldots, w_{m}\right)^{\top}$.

Using these discretizations, we can construct the one-form Dirichlet matrix

$$
L_{i j}=\int_{\Omega}\left(\nabla \eta_{i}\right):\left(\nabla \eta_{j}\right) \mathrm{d} x
$$

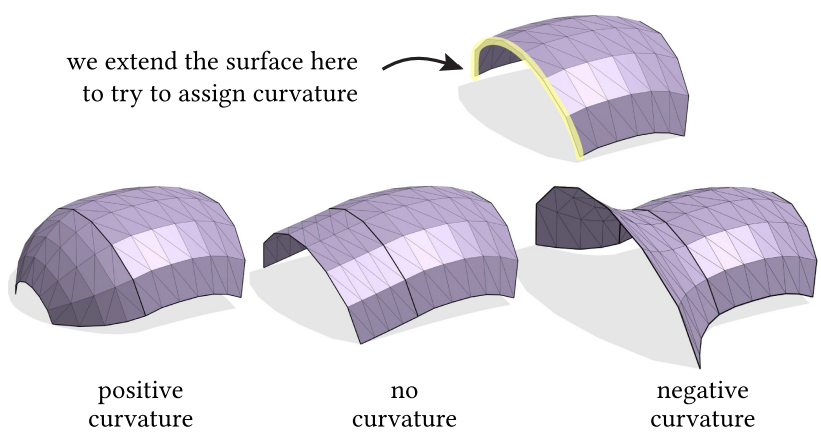

Fig. 6. For the boundary of a continuous, piecewise linear surface (top) there is no way to uniquely assign curvature at the boundary. The surface can be extended in many different ways that yield different curvatures at the boundary, examples leading to positive (bottom left), no (bottom center), and negative (bottom right) curvature are shown.

the differential matrix

$$
D_{i j}=\int_{\Omega} \eta_{i} \cdot \mathrm{d} \varphi_{j} \mathrm{~d} x
$$

the mass matrix

$$
M_{i j}=\int_{\Omega} \eta_{i} \cdot \eta_{j} \mathrm{~d} x
$$

and the curvature matrix

$$
K_{i j}=\int_{\Omega} \kappa \eta_{i} \cdot \eta_{j} \mathrm{~d} x
$$

The matrix entries are provided in Appendix A.

Using these matrices, we write the discrete version of Equation (26) as seeking a critical point of the expression

$$
\frac{1}{2} \mathbf{w}^{\top}(L+K) \mathbf{w}-\lambda^{\top}(M \mathbf{w}-D \mathbf{u}),
$$

for $\mathbf{u} \in \mathbb{R}^{n}, \mathbf{w}, \lambda \in \mathbb{R}^{m}$. Differentiating with respect to $\lambda$ gives $M \mathbf{w}=D \mathbf{u}$. As $M$ is invertible, we get the system

$$
\underset{\mathbf{u}}{\operatorname{argmin}} \mathbf{u}^{\boldsymbol{\top}} D^{\top} M^{-1}(L+K) M^{-1} D \mathbf{u} \text {. }
$$

This optimization problem can now be solved with a variety of constraints or mixed with other energy terms, depending on the application.

\subsection{Crouzeix-Raviart One-forms}

While there are multiple approaches to discretizing tangent oneforms for triangle meshes, we choose to base our approach on Crouzeix-Raviart finite elements (see Section 2 for a discussion). The advantage of this approach is its simplicity. Crouzeix-Raviart basis functions are only ever nonzero on two adjacent triangles, so every basis function lives on an intrinsically flat domain: The two triangles can be unfolded without distortion. This means that our discretization will account for curvature correctly in the end, without having to explicitly address issues like parallel transport during construction. 
6.2.1 Introduction to Crouzeix-Raviart. The scalar CrouzeixRaviart basis function for the edge $e_{i j}$ is defined to be 1 on the edge itself, -1 on the two vertices $k, l$ opposite the edge, and linear on the two triangles $T_{i j k}, T_{j i l}$ [Braess 2007, p. 109]. For boundary edges, only one triangle needs to be considered. As a result, it is 0 on the midpoints of the edges $e_{j k}, e_{k i}, e_{i l}, e_{l j}$ (see Figure 5, left). The scalar Crouzeix-Raviart element is not continuous, except at the midpoints of edges. This makes it a non-conforming element, and if it is used in a Galerkin method, one speaks of the discontinuous Galerkin method. Despite being nonconforming, it is known to converge for certain problems, most notably the Poisson equation in $\mathbb{R}^{2}$ [Braess 2007, III, Theorem 1.5].

6.2.2 One-forms. The scalar Crouzeix-Raviart element can be used to define a finite element space for one-forms. At the midpoint of every edge $e$ of a flat triangle pair, the space of one-forms is spanned by the two forms $\omega_{e}^{\|}, \omega_{e}^{\perp}$, such that

$$
\begin{aligned}
& \left\langle\omega_{e}^{\|}, \mathbf{t}_{e}\right\rangle=1, \quad\left\langle\omega_{e}^{\|}, \mathbf{n}_{e}\right\rangle=0, \\
& \left\langle\omega_{e}^{\perp}, \mathbf{t}_{e}\right\rangle=0, \quad\left\langle\omega_{e}^{\perp}, \mathbf{n}_{e}\right\rangle=1,
\end{aligned}
$$

where $\mathbf{n}_{e}$ is the (oriented) perpendicular vector of the edge $e$ in each triangle, $\mathbf{t}_{e}$ is the (oriented) tangent of the edge $e$, and the angle bracket $\langle\cdot, \cdot\rangle$ denotes the pairing of a form with a vector. See Figure 5 (right) for an illustration. The definition of $\omega_{e}^{\perp}$ depends on which triangle one is in, but only in an extrinsic way: In the intrinsic geometry of the triangle pair, the edge is completely flat, thus the two covectors $\omega_{e}^{\perp}$ defined in each triangle are the same covector intrinsically. Because of this, both $\omega_{e}^{\|}$and $\omega_{e}^{\perp}$ can be easily extended to the triangles adjacent to $e$ : Since the triangle pair (or the one triangle) is intrinsically flat, parallel transport along the triangles is trivial, and we can easily extend the definition of $\omega_{e}^{\perp}$ and $\omega_{e}^{\|}$to the interior of the triangles adjacent to $e$.

If $b_{e}$ is the Crouzeix-Raviart basis function for the edge $e$, then we define its two CROF basis function as

$$
\begin{aligned}
b_{e}^{\|} & :=\omega_{e}^{\|} b_{e}, \\
b_{e}^{\perp} & :=\omega_{e}^{\perp} b_{e} .
\end{aligned}
$$

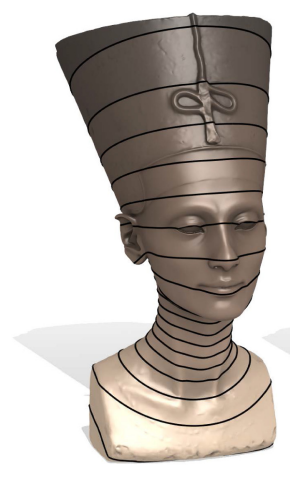

Laplacian energy
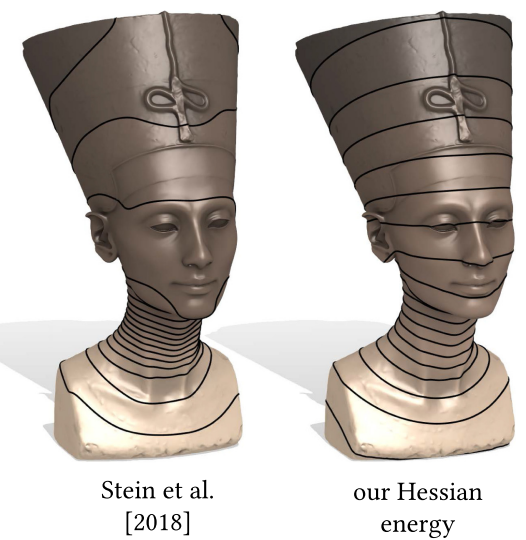

energy
Fig. 7. The first nonzero eigenvector of the Laplacian energy $E_{\Delta^{2}}$ (left), the Hessian of Stein et al. [2018] (center), and the curved Hessian energy $E$ (right). The eigenvectors of $E_{\Delta^{2}}$ and $E$ look similar, since they both discretize the biharmonic energy. The method of Stein et al. [2018] visibly disagrees.
Defined this way, CROFs have the correct notion of parallel transport without having to explicitly account for it. Consider a path $\gamma$ through all edge midpoints of edges emanating from a vertex $v$ in a counterclockwise direction (see inset). We start with a single tangent vector on the midpoint of one edge, corresponding to a combination of two basis functions, and see what angle we pick up when going around the vertex $v$ using our basis functions. We now go along the path $\gamma$, moving from edge-to-edge by choosing successive basis functions so the sum of the basis functions from two adjacent edges is constant on the shared triangle. Doing that corresponds exactly to parallel transport on a cone mani-

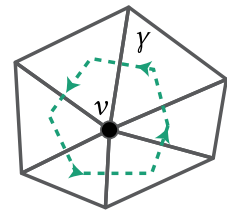
fold: The tangential part of the vector at each edge does not change extrinsically at all when crossing the edge along $\gamma$. The perpendicular basis function jumps extrinsically: The angle between normal vectors on each side of the edge is $\pi$ minus the dihedral angle of the edge. At the end of our journey along $\gamma$, when we are back at our original edge, our starting vector picked up angle defect corresponding to the discrete curvature of the mesh. The CROF basis functions have accounted for the discrete curvature of the mesh in the sense of curvature on cone manifolds [Wardetzky 2006] without having to explicitly account for parallel transport during the construction of the basis functions.

Since every basis function is only supported on at most two triangles, the matrices $L, M, D, K$ will be sparse. The matrix $M$ is diagonal, which makes it easy to invert. The matrix entries can be found in Appendix A.

6.2.3 The Curvature Term. Special care needs to be applied when computing the matrix $K$. The Gaussian curvature $\kappa$ of an intrinsically flat pair of triangles would appear, at first, to be 0 . But actually, the Gaussian curvature of a polyhedron is entirely concentrated on its vertices (and is zero anywhere else). The

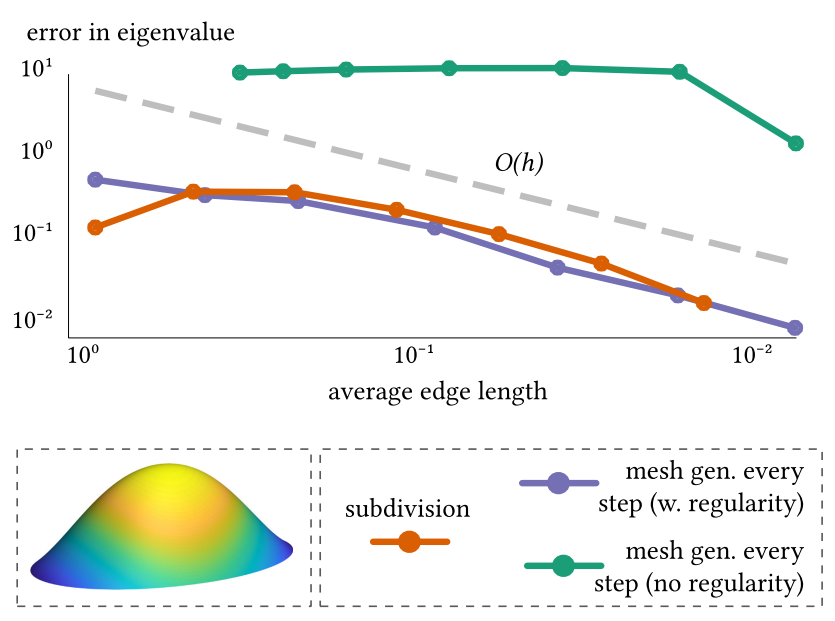

Fig. 8. Computing the fourth eigenvalue of the Hessian energy $E$ on an ellipse that was distorted in the third dimension (bottom left). Both refinement through Loop subdivision and projection to a given smooth surface, as well as generating a planar mesh of the desired resolution with regular triangles at every step and then projecting to a given smooth surface, show convergence to the highest resolution. For simple mesh generation without triangle regularity, no convergence is observed. 
integrated Gaussian curvature at a vertex is also known as the angle defect

$$
\kappa_{v}:=2 \pi-\sum_{f \in N(v)} \theta_{v}^{f},
$$

where the sum is over all faces $f$ in the set of faces containing the vertex $v$, and $\theta_{v}^{f}$ is the angle at vertex $v$ in face $f$ [Grinspun et al. 2006]. The idea of angle defects is very old: It goes back all the way to at least Descartes, c. 1630, who showed that the sum of all angle defects of a polyhedron with spherical topology is $4 \pi$ [Federico 1982].

We thus interpret the Gaussian curvature of the polyhedron as a collection of delta functions at every vertex, i.e.,

$$
\kappa:=\sum_{v} \kappa_{v} \delta_{v}
$$

where $\delta_{v}$ is the Dirac delta. This means that the integral of $\kappa g$, where $\kappa$ is the Gaussian curvature and $g$ is any continuous function over the triangle $T_{i j k}$ with vertices $i, j, k$, can be written as:

$$
\int_{\Omega} \kappa g \mathrm{~d} x=\sum_{v} \kappa_{v} g(v) .
$$

If the function $g$ itself is only continuous in each triangle, then we need to distribute the contribution of each triangle accordingly. Let $s_{v, f}>0$ for each vertex $v$ and each face $f$ in the neighborhood of $v$ be coefficients that average the contribution of each face at a vertex, i.e., the sum of the $s_{v, f}$ over all faces $f$ in the neighborhood of $v$ is one. Then,

$$
\int_{\Omega} \kappa g \mathrm{~d} x=\sum_{v} \kappa_{v} \sum_{f \in N(v)} s_{v, f} g_{f}(v),
$$

where $g_{f}$ is the function $g$ in the triangle $f$ and $N(v)$ is the set of all faces in the neighborhood of $v$. We choose to average by tip angle, which corresponds to an integral along a small circle around the vertex. We did not explore other reasonable choices, such as averaging by face area. This formula is used to compute the entries of $K$; they are given in Appendix A.

One remaining issue with the angle defect as Gaussian curvature is that the angle defect is not defined at boundary vertices. The problem stems from the fact that the notion of curvature at the boundary of meshes (continuous, piecewise linear surfaces) is not in and of itself meaningful: By choosing to extend the surface in different ways at the boundary, we can achieve any arbitrary Gaussian curvature, as can be seen in Figure 6. We choose to set the angle defect to 0 for all boundary vertices, thereby choosing the most developable (intrinsically linear) extension of all possible extensions. This fits in with our as-linear-as-possible boundary conditions but differs from some conventions of angle defect at the boundary, which define it as the sum of tip angles subtracted from $\pi$ (which is a discretization of geodesic curvature).

\subsection{Observed Numerical Convergence}

Using our CROF discretization of the Hessian energy to solve a variety of problems, we observe convergence on the order of the average edge length $h$ (Figure 9). As can be seen in Figure 8, a successful strategy for obtaining convergence is making sure that the vertices are contained in a smooth surface, and then either refining the mesh through Loop subdivision [Loop 1987] with a fixed smooth boundary or generating meshes that fulfill the triangle regularity condition: The ratio of circumcircle to incircle of each triangle (the triangle regularity) is bounded from above and below independent of refinement level. This condition is standard for finite elements [Braess 2007, Definition 5.1 (uniform triangulation)]. The order of convergence and the triangle regularity condition correspond to the discretization of the Laplacian energy with zero Neumann boundary conditions, $E_{\Delta^{2}}$, with mixed FEM in the flat setting [Jacobson et al. 2010; Scholz 1978]. However, we do not have a proof of convergence for our method to confirm this convergence rate.
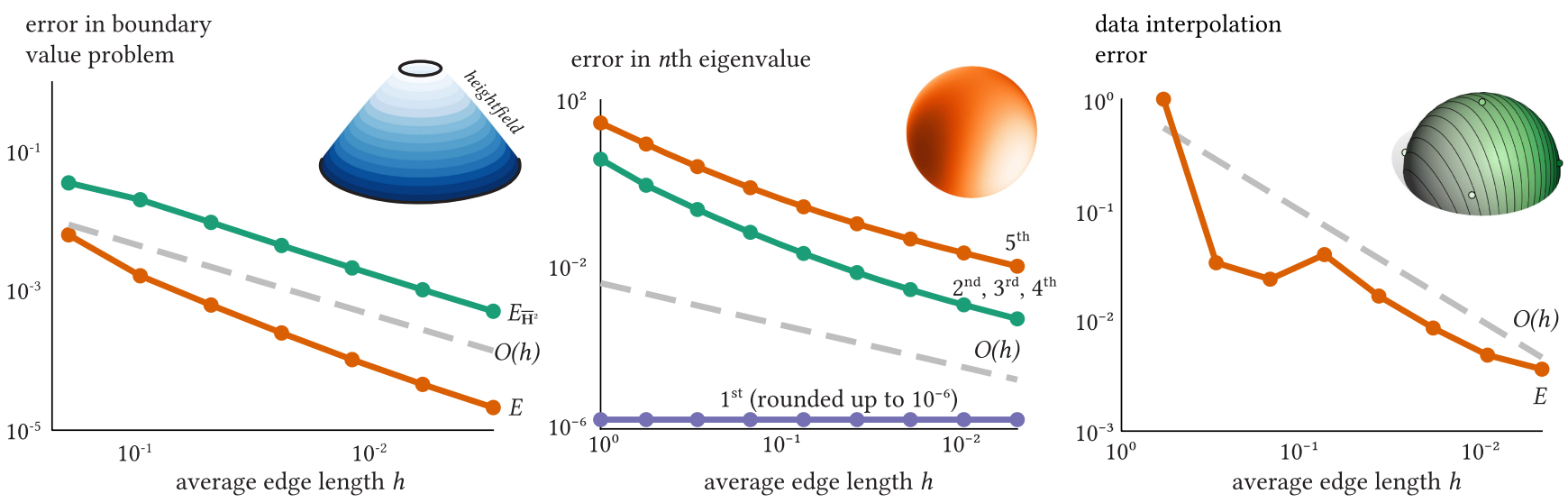

Fig. 9. Convergence plots for three different problems, all errors are $L^{2}$ errors. Boundary value problem with known exact solution on a flat annulus mesh refined by loop subdivision with fixed smooth boundary; both our Hessian $E$ and the planar Hessian $E_{\overline{\mathbf{H}}^{2}}$ of Stein et al. [2018] are shown (even though, for planar domains, the smooth curved and planar Hessian energies coincide, the different discretizations result in a different error) (left). Error in calculating the lowest eigenvalues of the operator associated with $E$ on the sphere with icosahedral meshing, with vertices of the mesh inscribed in the smooth limit sphere (center). Solving an interpolation problem and computing the error with respect to the highest-resolution solution, refined by loop subdivision with fixed $z$-coordinate at the boundary (right). 

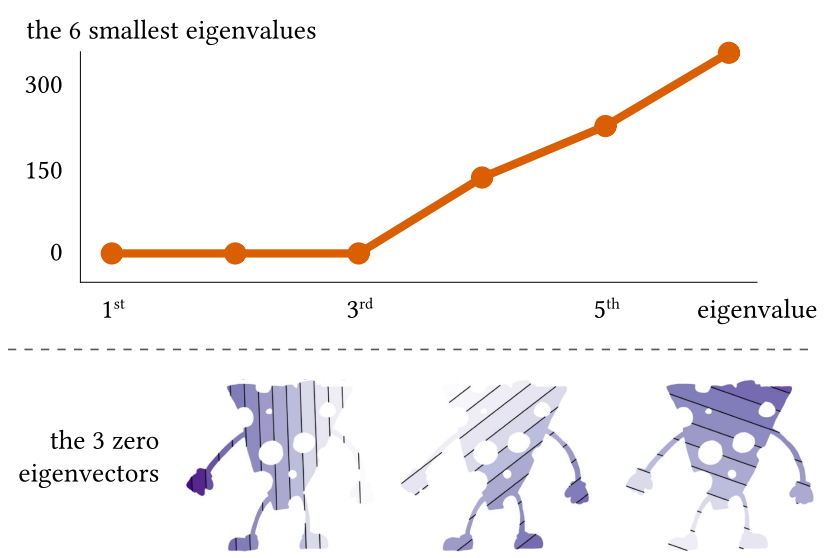

Fig. 10. The six lowest eigenvalues of the Hessian energy discretized with CROF on the cheeseman (top). As expected, there are only three zero eigenvalues. The three lowest eigenvectors (bottom) are the linear functions, which correspond to the smooth Hessian energy.

Our method correctly reproduces the first eigenvector of the Laplacian energy on closed surfaces in the experiment proposed by Stein et al. [2018, Section 5.3.1] on a refined mesh (Figure 7). As mentioned in Stein et al. [2018, Section 4.5], discretizations can sometimes exhibit spurious modes in the kernel of the energy, which lead to wrong solutions. We have not proved that this does not happen for our CROF discretization of the Hessian energy, but we have not observed it in our experiments (see Figure 10 for the cheeseman example domain mentioned in Stein et al. [2018, p. 7]).

Further experiments can be found in Appendix B: Figures 15 and 16 feature additional convergence experiments confirming the order of convergence, Figure 17 examines the dependence of the result on the mesh further, and Figure 18 compares our implementation of the Hessian energy with other Hessian energies in the flat case.

\section{APPLICATIONS}

We implement the optimization of Equation (27) by constructing a sparse matrix in C++ using Eigen [Guennebaud et al. 2010] and then manipulating and optimizing it in MATLAB [2019] with mex. For linear equality constraints, we use the optimizer of Jacobson et al. [2019a, min_quad_with_fixed] via the library of Jacobson [2019]. Using this approach, complicated constraints are also possible, such as linear and quadratic inequality constraints for more complicated applications. Since the Hessian energy is a quadratic energy, optimizers using the interior point method (such as the solver of Andersen and Andersen [2000]) are appropriate.

\subsection{Scattered Data Interpolation}

Like any smoothness energy, the Hessian energy can be used for scattered data interpolation. One solves the following minimization problem, for some given interpolation data $u\left(x_{i}\right)=f_{i}, i=$ $1, \ldots, n$

$$
\underset{u}{\operatorname{argmin}} E(u) \quad u\left(x_{i}\right)=f_{i}, i=1, \ldots, n .
$$

As long as at least three interpolation points are provided, this problem has a solution. This is because the null space of the

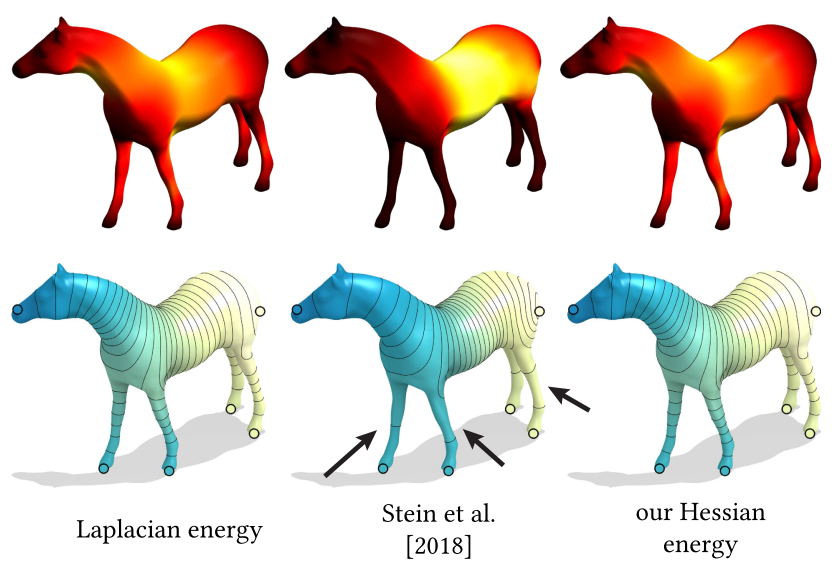

Fig. 11. Scattered data interpolation problem solved on a closed surface (bottom row) and the gradient of the solution (top row). $E_{\Delta^{2}}$ (left) provides a satisfying result-isolines are relatively evenly spaced, and the gradient is uniform. Stein et al. [2018] (center) has large variation in isoline distance (see arrows), and the gradient of the solution is less uniform. E (right) replicates the behavior of $E_{\Delta^{2}}$.

Hessian energy can have at most all linear functions in it, which is a three-dimensional space, and the null space of the Laplacian energy with zero Neumann boundary conditions contains only constant functions, which is a one-dimensional space [Stein et al. 2018].

The choice of smoothness energy will greatly influence the quality of the result. The Laplacian energy with zero Neumann boundary conditions, $E_{\Delta^{2}}$, is a popular method, since it produces smooth, evenly spaced isolines, which results in natural-looking interpolation and extrapolation. This is because the gradient of the solution is relatively uniform across the surface. As can be seen in Figure 11, our curved Hessian energy $E$ reproduces the desirable behavior of the Laplacian energy for surfaces without boundary. The implementation of the planar Hessian energy $E_{\overline{\mathbf{H}}^{2}}$ for curved surfaces by Stein et al. [2018] fails to do so: The distance between the isolines varies greatly, for example on the legs. The isolines also experience significant bunching at the rump and back of the horse.

However, the Laplacian energy is known to produce bias near domain boundaries due to its low-order boundary conditions: Isolines of solutions bend so they can be perpendicular to the boundary. This was one of the motivations of Stein et al. [2018], and thus their planar Hessian energy minimizes the influence of the boundary by employing natural boundary conditions that make the function as-linear-as-possible. Figure 4 shows that our Hessian energy $E$ does not show the bias at the boundary that the Laplacian energy does: this is because it also has as-linear-as-possible natural boundary conditions.

For this application, our Hessian energy $E$ combines the two worlds of Laplacian energy and planar Hessian energy to produce a smoothness energy that is suited for scattered data interpolation on curved surfaces while unbiased by the presence of boundaries (Figure 1, Figure 12). This is helpful if the boundaries of the surface do not have any physical meaning: Perhaps they are the result of a faulty laser scan, or perhaps surface information is simply not available there. The Hessian energy's natural boundary conditions 


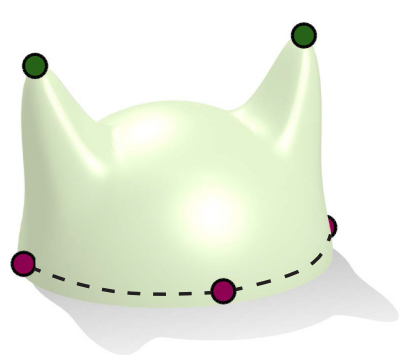

data to interpolate

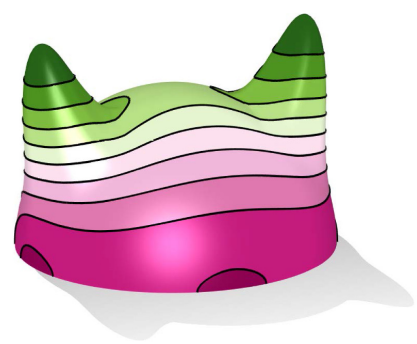

Laplacian energy (zero Neumann boundary conditions)

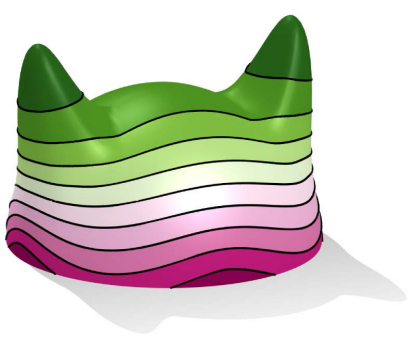

Stein et al. [2018]

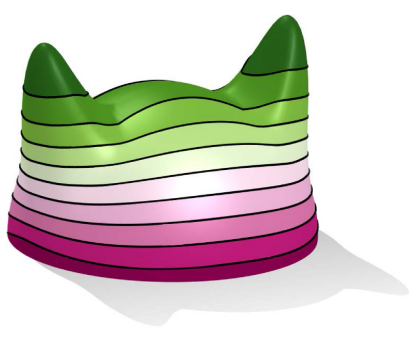

our Hessian energy

Fig. 12. Solving an interpolation problem on a Viking helmet. Our goal here is to preserve the dashed line (which is almost a geodesic) connecting three data points of the same value (far left). Using $E_{\Delta^{2}}$ distorts the line near the boundary, since the zero Neumann boundary conditions make the isolines perpendicular to the boundary (center left). Using the planar Hessian of Stein et al. [2018] still leads to some distortion due to not accounting for the surface's curvature (center right). Our Hessian energy $E$ correctly accounts for the curvature of the surface and does not suffer from bias at the boundary, interpolating the dashed line as desired (far right).

make a best guess everywhere the data are missing by extrapolating the function linearly across the boundary.

\subsection{Data Smoothing}

Another popular application for smoothness energies is the eponymous data smoothing. This can be used to simply smooth arbitrary data, to denoise noisy data, or to smooth the surface itself via surface fairing. One solves the following Helmholtz-like optimization problem: given an input function $f$ to be smoothed,

$$
u=\underset{u}{\operatorname{argmin}} E(u)+\alpha \int_{\Omega}(f-u)^{2} \mathrm{~d} x,
$$

where the parameter $\alpha>0$ is a trade-off between the input data and the smoothness of the output data.

Figure 2 shows our Hessian energy $E$ applied to such a smoothing problem. Correctly accounting for curvature by modeling a curved biharmonic equation using the Laplace-Beltrami operator is important here: The figure shows that the approach of Stein et al. [2018] causes distortion in high-curvature regions when smoothing a step function. In this figure the smoothing parameters are chosen to give visually similar amounts of smoothing, which means a slightly larger parameter $\alpha$ for the method of Stein et al. [2018].

It is natural to ask why the fact that minimizers of $E_{\overline{\mathbf{H}}^{2}}$ do not solve the biharmonic equation leads to worse results when smoothing the step function of Figure 2, but not for the smoothing problems solved by Stein et al. [2018, Figure 1, Figure 11, Figure 13]. These examples all smooth very noisy functions with a lot of variation everywhere on the surface. The step function is the opposite of that: the variation is much more sparse. This allows the error of not accounting for curvature correctly to manifest. In Figure 13 such a denoising problem is solved using the energies $E_{\Delta^{2}}$ (with zero Neumann boundary conditions), $E_{\overline{\mathbf{H}}^{2}}$ (with the implementation of Stein et al. [2018]), and $E$. It can be clearly seen that $E_{\Delta^{2}}$, the Laplacian energy with zero Neumann boundary conditions, is biased by the boundary, and the isolines near the boundary are distorted so they can be normal to it. The denoised solution using the Hessian energy $E$ does not suffer from this, and the isolines ignore the boundary. In regions far away from the boundary it can be observed that the result of denoising with the Hessian energy $E$ matches the Laplacian energy with zero Neumann boundary conditions $E_{\Delta^{2}}$, while the planar Hessian energy $E_{\overline{\mathbf{H}}^{2}}$ differs.

The smoothing problem can also be used to smooth the geometry of the surface itself if the input data $f$ from Equation (34) is the vertex positions in each coordinate, and the output data $u$ is the new vertex positions. If such a smoothing operation is applied repeatedly, then one has a smoothing flow. Figure 14 shows our Hessian energy $E$ applied to such a problem. While the method of Stein et al. [2018] can lead to some artifacts due to not accounting for curvature, this does not happen with our curved Hessian energy $E$.

\section{CONCLUSION}

In this work, we have introduced a smoothing energy for curved surfaces, the Hessian energy. Its minimizers solve the biharmonic equation, and it exhibits the as-linear-as-possible natural boundary conditions in the curved setting that the planar Hessian energy of Stein et al. [2018] exhibits in the flat setting. This Hessian energy can be used in many applications where smoothness energies are required, these smoothness energies should be unbiased by the boundary, and it is crucial that the minimizers of the energy solve the biharmonic equation.

\subsection{Limitations}

We have no numerical analysis proof for the convergence of our discretization method. We also do not provide any theoretical analysis of the spectrum of our discrete operator. Both are needed to make this discretization reliable and to improve understanding of the method, where it works, and where it does not.

\subsection{Future Work}

One interesting avenue for future work is to explore alternate discretizations. Higher-order versions of Crouzeix-Raviart basis functions, such as cubic or quintic basis functions, would be an interesting potential improvement. Alternatively, instead of choosing the intermediate variable $w=\mathrm{d} v$ for the mixed formulation as in Equation (25), a discretization where $w=\nabla \mathrm{d} v$ sounds very 


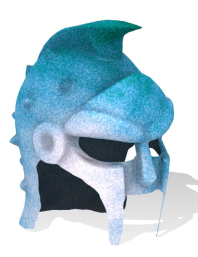

noisy function

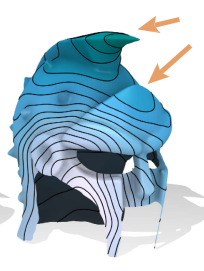

Stein et al. [2018]

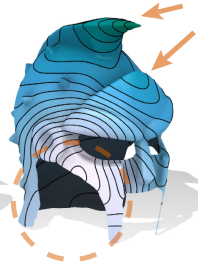

Laplacian energy zero Neumann

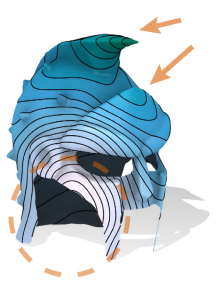

our Hessian energy
Fig. 13. Denoising a function (far left) via smoothing. The Hessian energy $E$ (far right) does not show the bias at the boundary that the Laplacian energy with zero Neumann boundary conditions $E_{\Delta^{2}}$ (center right) does, indicated by the orange circle. Away from the boundary, the results for $E$ and $E_{\Delta^{2}}$ agree, while the method of Stein et al. [2018] (center left) differs, indicated by the orange arrows.

promising. This would more closely mirror the mixed formulation of Stein et al. [2018]. The CROF approach can be used to define a basis for tensors in the same way as is done for vectors in Section 6.2, based on the parallel and the perpendicular vector at each edge. Using other finite elements to discretize the space of oneforms could also produce new methods. Moreover, future work could explore discretizations of the smooth energy on other surface representations beyond triangle meshes.

A rich source of future work is the numerical analysis of our method. We do not have any proof of convergence or a solid mathematical analysis of the spectrum of our operator, and while the experiments in Section 6.3 provide some evidence for problems that can be solved with our discretization of $E$, a thorough numerical analysis treatment of our discretization would be valuable to exactly identify the strengths and weaknesses of our method. Our Crouzeix-Raviart discretization is a potential candidate for spurious modes, since the finite element is non-conforming, even though we have not observed them in practice. The method of English and Bridson [2008] is an example of a Crouzeix-Raviart discretization that works for many cases, but where specific triangle configurations exist that lead to spurious modes [Quaglino 2012, Section 4.4.2]. The properties of minimizers of the discrete energies also warrant further investigation: It is unclear which properties of smooth minimizers they actually inherit and which properties only hold in the limit.

Another interesting direction for future work is to consider additional applications. Smoothness energies have many uses, and if such an application has to be unbiased by the boundary even on heavily curved surfaces, our Hessian energy $E$ is a powerful tool. Applications could include animation [Jacobson et al. 2011], distance computation [Crane et al. 2013b], and more.

Moreover, our simple Crouzeix-Raviart discretization of the one-form Dirichlet energy containing covariant derivatives from Section 6.2 offers an interesting approach to discretize the vector Dirichlet energy in a wide variety of applications. Potential applications include vector field design [Knöppel et al. 2013], parallel transport of vectors [Sharp et al. 2018], and many more [Azencot et al. 2015; Corman and Ovsjanikov 2019; Liu et al. 2016].

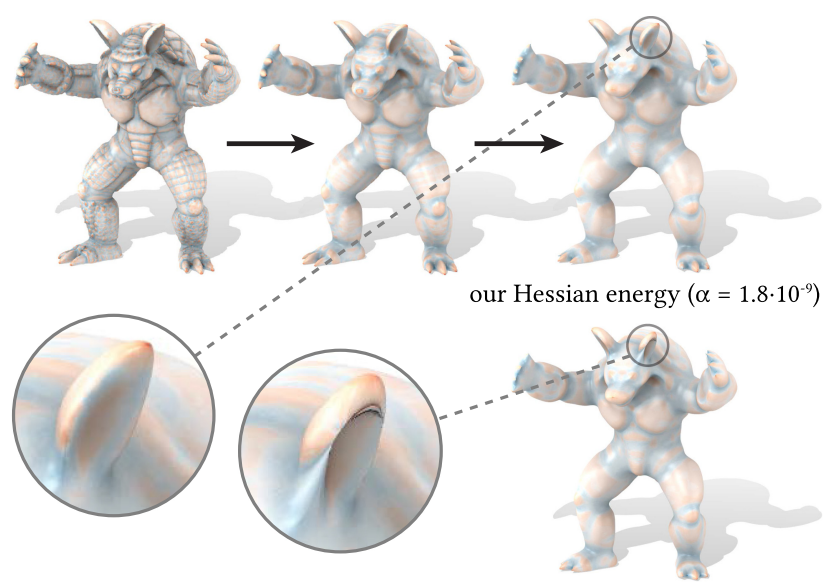

Stein et al. [2018] $\left(\alpha=2.5 \cdot 10^{-9}\right)$

Fig. 14. Smoothing flow for an armadillo. The surfaces are colored by angle defect. Each step of our Hessian energy $E$ (top) leads to a smoother result. Smoothing with Stein et al. [2018] (bottom) can lead to artifacts in regions with curvature, such as the highlighted ears. The smoothing parameter $\alpha$ was chosen to produce a similar amount of smoothing in both methods. Three smoothing steps were computed.

\section{APPENDIX}

\section{A IMPLEMENTATION}

The entries for each of the matrices defined in Section 6.1 needed to construct the system matrices used in Equation (27) are as follows: Let $e$ be an oriented edge from the vertex $i$ to $j$. The two triangles adjacent to $e$ are $T_{i j k}$ and $T_{j i l}$, and $f$ is an oriented edge from the vertex $k$ to $i$. The entries of the symmetric CROF vector Dirichlet matrix $L$ on the triangle $T_{i j k}$ are

$$
\begin{gathered}
L_{e^{\|}, e^{\|}}^{i j k}=L_{e^{\perp}, e^{\perp}}^{i j k}=\frac{2}{A_{i j k}}, \\
L_{e^{\|,} e^{\perp}}^{i j k}=0, \\
L_{e^{\|}, f^{\|}}^{i j k}=L_{e^{\perp}, f^{\perp}}^{i j k}=\frac{2}{A_{i j k}} \cos ^{2} \theta_{i}^{i j k}, \\
L_{e^{\perp}, f^{\|}}^{i j k}=-L_{e^{\|,}, f^{\perp}}^{i j k}=\frac{2}{l_{i j} l_{k i}} \cos \theta_{i}^{i j k},
\end{gathered}
$$

where $A_{i j k}$ is the double area of the triangle $T_{i j k}, \theta_{i}^{i j k}$ is the angle in the triangle $T_{i j k}$ at the vertex $i$, and $l_{i j}$ is the length of the edge from vertex $i$ to $j$. If one of the edges has reversed orientation in the triangle $T_{i j k}$ with respect to its global orientation, then its offdiagonal entries get multiplied by -1 . These are only the terms for the triangle $T_{i j k}$. One must add the terms for all triangles and all pairs of edges in that triangle to compute the full matrix $L$. We suggest looping through all triangles and adding the terms for each triangle to the respective entries of the matrix corresponding to the edges. This can easily be parallelized with a parallel_for loop.

The entries of the diagonal CROF mass matrix $M$ on the triangle $T_{i j k}$ are

$$
M_{e^{\|}, e^{\|}}^{i j k}=M_{e^{\perp}, e^{\perp}}=\frac{A_{i j k}}{6 l_{i j}^{2}} .
$$



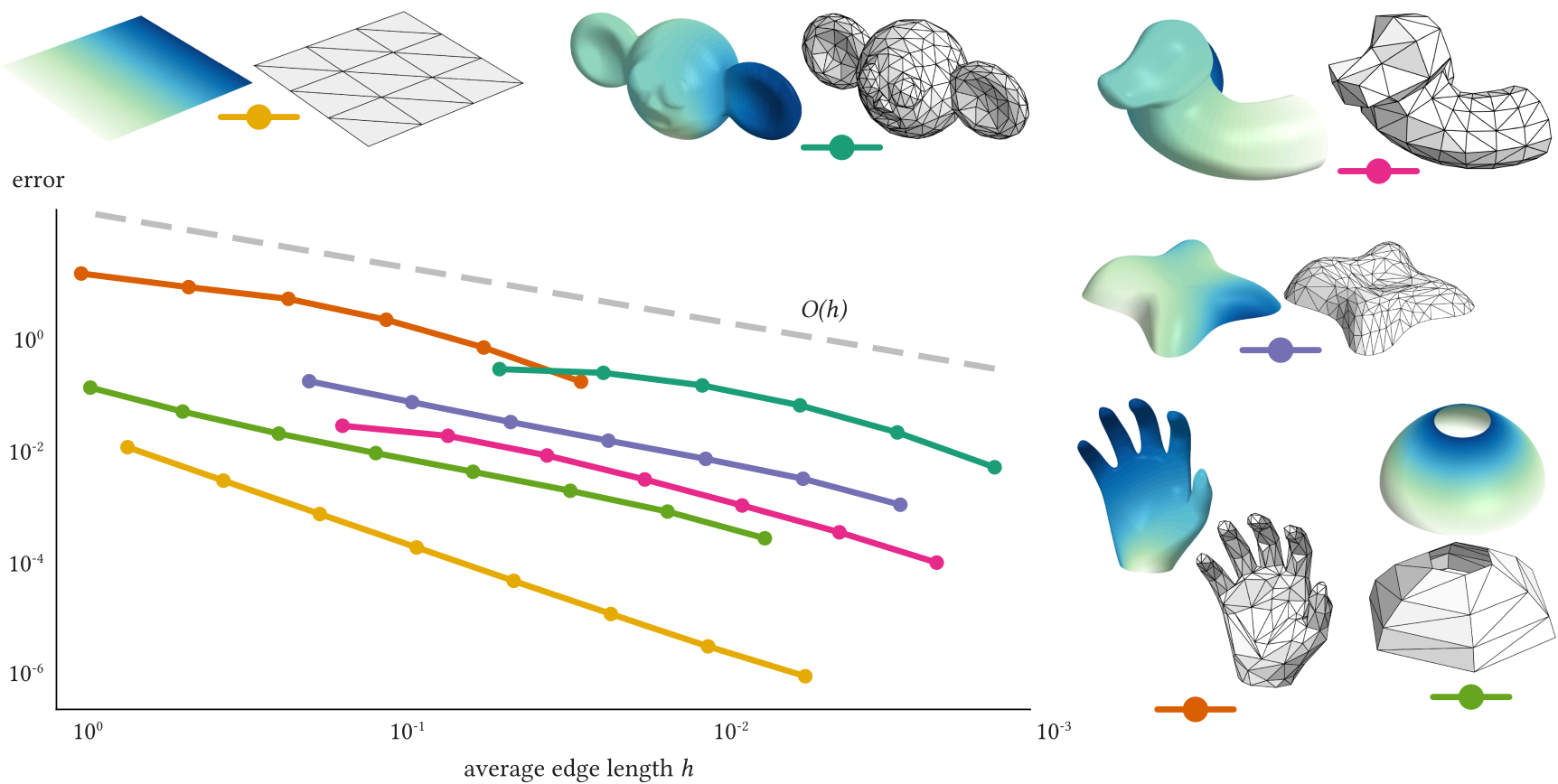

Fig. 15. Error plot for six different boundary value problems. The minimizer of the Hessian energy $E$ discretized with our discretization is compared to a high-resolution solution with the same discretization. Refinement happens via loop subdivision with various types of fixed boundary. The high-resolution solution as well as the wireframe of the lowest-resolution mesh are displayed for each problem.
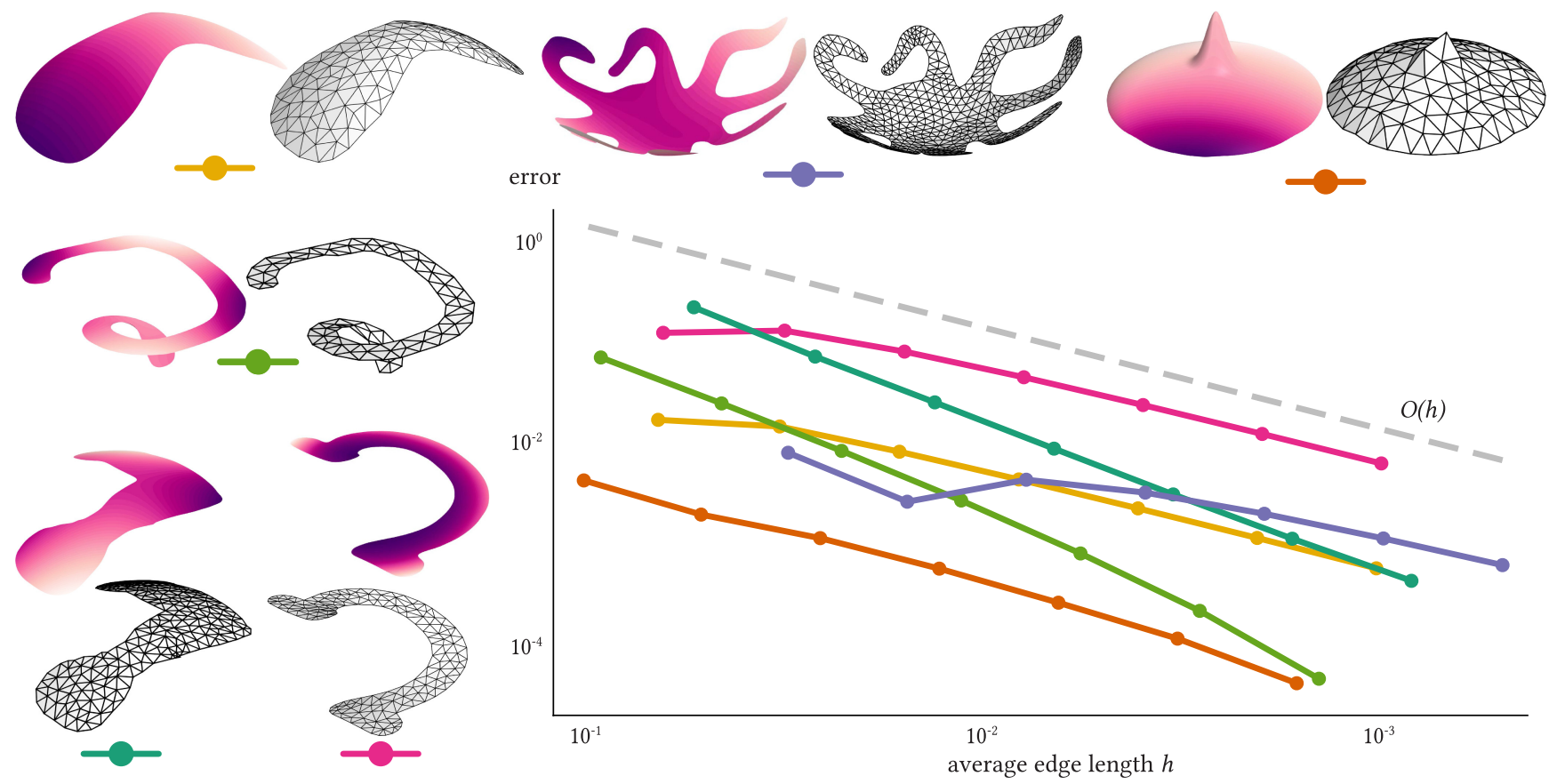

Fig. 16. Error plot for six different forward problems. The domains are curved surfaces of the form $(x, y, z(x, y)) \in \mathbb{R}^{3}$, so the integrand of the Hessian energy can be exactly computed pointwise using the properties of Monge patches [Weisstein 2019]. Quadrature is then used to compute the exact value of $E(f)$. The high-resolution function $f$ as well as the wireframe of the lowest-resolution mesh are displayed for each problem. Refinement happens via loop subdivision, and then projection to the given smooth surface. 

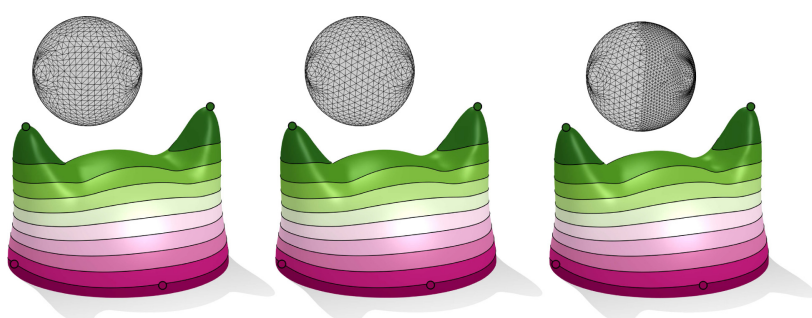

Fig. 17. The same scattered data interpolation problem solved on different meshes for surfaces similar to the one from Figure 12 using the Hessian energy $E$. The results are very similar. The wireframe shows each of the meshes before further refinement through loop subdivision with fixed boundary.

The entries of the differential matrix $D$ on the triangle $T_{i j k}$ for each edge $e$ are

$$
\begin{aligned}
-D_{i, e^{\|}}^{i j k}=D_{j, e^{\|}}^{i j k} & =\frac{A_{i j k}}{6 l_{i j}^{2}}, \\
D_{k, e^{\|}}^{i j k} & =0, \\
D_{i, e^{\perp}}^{i j k} & =-\frac{l_{j k}}{6 l_{i j}} \cos \theta_{j}^{i j k}, \\
D_{j, e^{\perp}}^{i j k} & =-\frac{l_{k i}}{6 l_{i j}} \cos \theta_{i}^{i j k}, \\
D_{k, e^{\perp}}^{i j k} & =\frac{1}{6},
\end{aligned}
$$

where $i$ is the vertex at the tail of the edge $e$, and $j$ is at its tip. If one of the edges has reversed orientation in the triangle $T_{i j k}$ with respect to its global orientation, then its entries get multiplied by -1 .

The entries of the curvature correction matrix $K$ on the triangle $T_{i j k}$ are

$$
\begin{aligned}
& K_{e^{\|,}, e^{\|}}^{i j k}=K_{e^{\perp}, e^{\perp}}^{i j k}=\frac{1}{l_{i j}^{2}}\left(\frac{\theta_{i}^{i j k}}{s_{i}} \kappa_{i}+\frac{\theta_{j}^{i j k}}{s_{j}} \kappa_{j}+\frac{\theta_{k}^{i j k}}{s_{k}} \kappa_{k}\right), \\
& K_{e^{\|}, e^{\perp}}^{i j k}=0, \\
& K_{e^{\|}, f^{\|}}^{i j k}=K_{e^{\perp}, f^{\perp}}^{i j k}=\frac{\cos \theta_{i}^{i j k}}{l_{i j} l_{k i}}\left(\frac{\theta_{j}^{i j k}}{s_{j}} \kappa_{j}+\frac{\theta_{k}^{i j k}}{s_{k}} \kappa_{k}-\frac{\theta_{i}^{i j k}}{s_{i}} \kappa_{i}\right), \\
& -K_{e^{\|}, f^{\perp}}=K_{e^{\perp}, f \|}=\frac{\sin \theta_{i}^{i j k}}{l_{i j} l_{k i}}\left(\frac{\theta_{j}^{i j k}}{s_{j}} \kappa_{j}+\frac{\theta_{k}^{i j k}}{s_{k}} \kappa_{k}-\frac{\theta_{i}^{i j k}}{s_{i}} \kappa_{i}\right) \text {, }
\end{aligned}
$$

where $\kappa_{v}$ is the angle defect at the vertex $v$ and $s_{v}$ is the angle sum at the vertex $v$. If one of the edges has reversed orientation in the triangle $T_{i j k}$ with respect to its global orientation, then its off-diagonal entries get multiplied by -1 .

\section{B ADDITIONAL EXPERIMENTS}

Figure 15 features a series of convergence experiments that shows the convergence of a boundary value problem on a variety of meshes to the highest-resolution solutions. In Figure 16, a series of
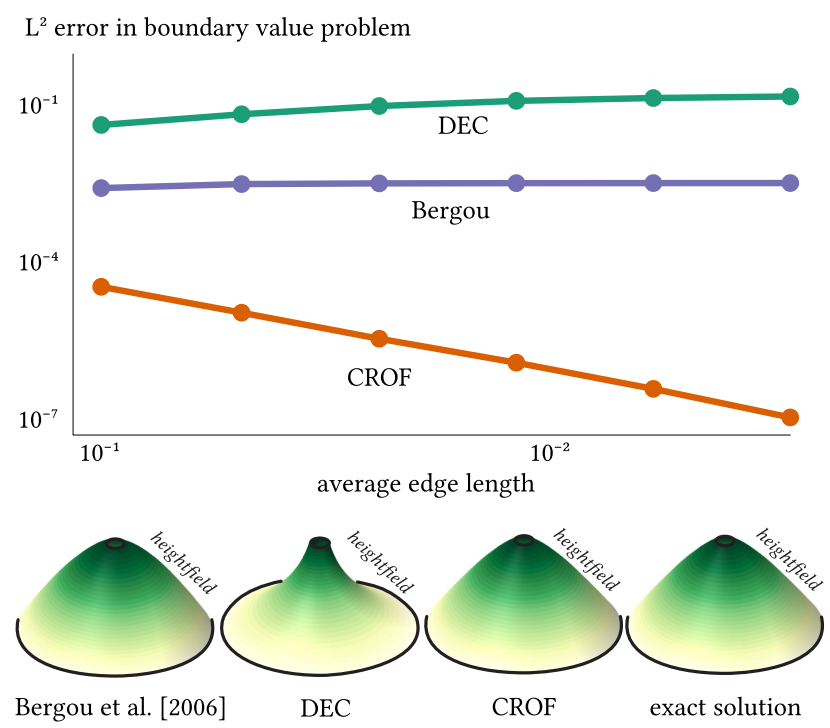

Fig. 18. A comparison of the CROF Hessian, the DEC Hessian (as of Stein et al. [2018, (20)], described by Fisher et al. [2007], and implemented by Wang et al. [2015]), and the Bergou Hessian (as of Stein et al. [2018, (21)], described by Bergou et al. [2006], and implemented by Wang et al. [2017]) in green. The two non-CROF Hessians fail to match the exact solution on the annulus, even though the method of Bergou et al. [2006] looks visually similar.

forward problems is solved, where the Hessian energy of a function is measured on a curved surface, and because both the function and the surface embedding are known, the exact solution is also known. This is used to measure the error. In both these examples, convergence of the order of the average edge length is observed.

Figure 17 shows that for different meshings of the same surface, very similar results are achieved, and the method is thus robust to remeshing. In Figure 18 our CROF implementation of the Hessian energy is compared with various Hessian energies discussed by Stein et al. [2018] in the flat annulus setting, where the exact solution is known.

\section{ACKNOWLEDGMENTS}

We thank the libigl data repository [Jacobson et al. 2019b] (horse, camel, cross lilium, hand, puppet head by Cosmic blobs), the Stanford 3D Scanning Repository [Graphics 2014] (armadillo), Crane [2018] (rubber duck, man-bridge, spot the cow, Nefertiti by Nora Al-Badri and Jan Nikolai Nelles), Jacobson [2013] (various cartoons in Figure 16), YahooJAPAN [2013] (plane), jansentee3d [2018] (tower), and Javo [2016] (helmet) for some of the meshes used in this work.

We thank Anne Fleming, Henrique Maia, and Peter Chen for proofreading.

\section{REFERENCES}

E. D. Andersen and K. D. Andersen. 2000. The MoseK interior point optimizer for linear programming: An implementation of the homogeneous algorithm. In High Performance Optimization. Kluwer Academic Publishers, 197-232.

Omri Azencot, Maks Ovsjanikov, Frédéric Chazal, and Mirela Ben-Chen. 2015. Discrete derivatives of vector fields on surfaces-an operator approach. ACM Trans. Graph. 34, 3 (May 2015), 29:1-29:13. 
Ilya Baran and Jovan Popovic. 2007. Automatic rigging and animation of 3D characters. ACM Trans. Graph. 26, 3 (July 2007), 72-es.

Miklos Bergou, Max Wardetzky, David Harmon, Denis Zorin, and Eitan Grinspun. 2006. A quadratic bending model for inextensible surfaces. In Proceedings of the 4th Eurographics Symposium on Geometry Processing. 227-230.

Miklós Bergou, Max Wardetzky, Stephen Robinson, Basile Audoly, and Eitan Grinspun. 2008. Discrete elastic rods. ACM Trans. Graph. 27, 3 (Aug. 2008), 63:1-63:12.

S. Bochner. 1946. Vector fields and Ricci curvature. Bull. Amer. Math. Soc. 52, 9 (1946), 776-797.

Daniele Boffi, Franco Brezzi, and Michel Fortin. 2013. Mixed Finite Element Methods and Applications. Springer-Verlag Berlin.

Mario Botsch and Leif Kobbelt. 2004. An intuitive framework for real-time freeform modeling. ACM Trans. Graph. 23, 3 (Aug. 2004), 630-634.

Dietrich Braess. 2007. Finite Elements (3rd ed.). Cambridge University Press.

Christopher Brandt, Leonardo Scandolo, Elmar Eisemann, and Klaus Hildebrandt. 2018. Modeling n-symmetry vector fields using higher-order energies. ACM Trans. Graph. 37, 2 (Mar. 2018), 18:1-18:18.

Susanne C. Brenner. 2015. Forty years of the Crouzeix-Raviart element. Numer. Meth Part. Differ. Equat. 31, 2 (2015), 367-396.

Etienne Corman and Maks Ovsjanikov. 2019. Functional characterization of deformation fields. ACM Trans. Graph. 38, 1 (Jan. 2019), 8:1-8:19.

R. Courant and D. Hilbert. 1924. Methoden der Mathematischen Physik-Erster Band Julius Springer, Berlin.

Keenan Crane. 2018. Keenan's 3D Model Repository. Retrieved from https://www.cs. cmu.edu/kmcrane/Projects/ModelRepository/.

Keenan Crane, Mathieu Desbrun, and Peter Schröder. 2010. Trivial connections on discrete surfaces. Comput. Graph. For. 29, 5 (2010), 1525-1533.

Keenan Crane, Ulrich Pinkall, and Peter Schröder. 2013a. Robust fairing via conformal curvature flow. ACM Trans. Graph. 32, 4 (July 2013), Article 61,10 pages.

Keenan Crane, Clarisse Weischedel, and Max Wardetzky. 2013b. Geodesics in heat: A new approach to computing distance based on heat flow. ACM Trans. Graph. 32 , 5 (Oct. 2013), 152:1-152:11.

Michel Crouzeix and P.-A. Raviart. 1973. Conforming and nonconforming finite element methods for solving the stationary Stokes equations I. ESAIM: Math. Modell. Numer. Anal.-Modél. Math. Anal. Numér. 7, R3 (1973), 33-75.

Mathieu Desbrun, Mark Meyer, Peter Schröder, and Alan H. Barr. 1999. Implicit fairing of irregular meshes using diffusion and curvature flow. In Proceedings of the 26th Conference on Computer Graphics and Interactive Techniques (SIGGRAPH'99). ACM Press/Addison-Wesley Publishing Co., New York, NY, 317-324.

Stephan Didas, Joachim Weickert, and Bernhard Burgeth. 2009. Properties of higher order nonlinear diffusion filtering. 7. Math. Imag. Vis. 35, 3 (2009), 208-226.

David L. Donoho and Carrie Grimes. 2003. Hessian eigenmaps: Locally linear embedding techniques for high-dimensional data. Proc. Nat. Acad. Sci. 100, 10 (2003), 5591-5596. DOI : https://doi.org/10.1073/pnas.1031596100

R. J. Duffin. 1959. Distributed and lumped networks. F. Math. Mech. 8,5 (1959), 793826.

Gerhard Dziuk. 1988. Finite Elements for the Beltrami Operator on Arbitrary Surfaces. Springer Berlin, 142-155

Elliot English and Robert Bridson. 2008. Animating developable surfaces using nonconforming elements. ACM Trans. Graph. 27, 3 (Aug. 2008), 66:1-66:5.

Lawrence C. Evans. 2010. Partial Differential Equations: (2nd ed.). American Mathematical Society.

P. J. Federico. 1982. Descartes on Polyhedra. Springer-Verlag New York Inc

Matthew Fisher, Peter Schröder, Mathieu Desbrun, and Hugues Hoppe. 2007. Design of tangent vector fields. ACM Trans. Graph. 26, 3 (2007).

Filippo Gazzola, Hans-Christoph Grunau, and Guido Sweers. 2010. Polyharmonic Boundary Value Problems. Springer-Verlag Berlin.

Todor Georgiev. 2004. Photoshop healing brush: A tool for seamless cloning. In Proceedings of the European Conference on Computer Vision.

Stanford Graphics. 2014. The Stanford 3D Scanning Repository. Retrieved from http:// graphics.stanford.edu/data/3Dscanrep/.

Eitan Grinspun, Mathieu Desbrun, Konrad Polthier, Peter Schröder, and Ari Stern. 2006. Discrete differential geometry: An applied introduction. In Proceedings of the ACM SIGGRAPH 2006 Courses (SIGGRAPH'06). ACM, New York, NY.

Gaël Guennebaud, Benoît Jacob et al. 2010. Eigen v3. Retrieved from http://eigen. tuxfamily.org.

Klaus Hildebrandt, Konrad Polthier, and Max Wardetzky. 2006. On the convergence of metric and geometric properties of polyhedral surfaces. Geom. Dedic. 123, 1 (Dec. 2006), 89-112.

Alec Jacobson. 2013. Algorithms and Interfaces for Real-time Deformation of 2D and 3D Shapes. Ph.D. Dissertation. ETH Zürich.

Alec Jacobson. 2019. gptoolbox-Geometry Processing Toolbox. Retrieved from https://github.com/alecjacobson/gptoolbox.

Alec Jacobson, Ilya Baran, Jovan Popović, and Olga Sorkine. 2011. Bounded biharmonic weights for real-time deformation. ACM Trans. Graph. 30, 4 (July 2011), 78:1-78:8.

Alec Jacobson, Daniele Panozzo et al. 2019a. libigl: A Simple C++ Geometry Processing Library. Retrieved from http://libigl.github.io/libigl/.
Alec Jacobson, Daniele Panozzo et al. 2019b. Libigl Tutorial Data. Retrieved from https: //github.com/libigl/libigl-tutorial-data.

Alec Jacobson, Elif Tosun, Olga Sorkine, and Denis Zorin. 2010. Mixed finite elements for variational surface modeling. Comput. Graph. For. 29, 5 (2010), 1565-1574.

Alec Jacobson, Tino Weinkauf, and Olga Sorkine. 2012. Smooth shape-aware functions with controlled extrema. Comput. Graph. For. 31, 5 (Aug. 2012), 15771586

jansentee3d. 2018. Dragon Tower. Retrieved from https://www.thingiverse.com/ thing:3155868.

Javo. 2016. Big Gladiator Helmet. Retrieved from https://www.thingiverse.com/thing: 1345281.

Pushkar Joshi, Mark Meyer, Tony DeRose, Brian Green, and Tom Sanocki. 2007. Harmonic coordinates for character articulation. ACM Trans. Graph. 26, 3 (July 2007), 71-es.

Jürgen Jost. 2011. Riemannian Geometry and Geometric Analysis. Springer-Verlag Berlin.

Kwang I. Kim, Florian Steinke, and Matthias Hein. 2009. Semi-supervised regression using Hessian energy with an application to semi-supervised dimensionality reduction. In Advances in Neural Information Processing Systems 22, Y. Bengio, D. Schuurmans, J. D. Lafferty, C. K. I. Williams, and A. Culotta (Eds.). Curran Associates, Inc., 979-987.

Felix Knöppel, Keenan Crane, Ulrich Pinkall, and Peter Schröder. 2013. Globally optimal direction fields. ACM Trans. Graph. 32, 4 (July 2013), 59:1-59:10.

Felix Knöppel, Keenan Crane, Ulrich Pinkall, and Peter Schröder. 2015. Stripe patterns on surfaces. ACM Trans. Graph. 34, 4 (July 2015), 39:1-39:11.

John M. Lee. 1997. Riemannian Manifolds. Springer-Verlag New York, Inc.

Stamatis Lefkimmiatis, Aurélien Bourquard, and Michael Unser. 2011. Hessian-based norm regularization for image restoration with biomedical applications. IEEE Trans. Image Proc. 21 (09 2011), 983-95.

Anat Levin, Dani Lischinski, and Yair Weiss. 2004. Colorization using optimization. In ACM SIGGRAPH 2004 Papers (SIGGRAPH '04). Association for Computing Machinery, New York, NY, 689-694.

Yaron Lipman, Raif M. Rustamov, and Thomas A. Funkhouser. 2010. Biharmonic distance. ACM Trans. Graph. 29, 3 (July 2010), 27:1-27:11.

Beibei Liu, Yiying Tong, Fernando De Goes, and Mathieu Desbrun. 2016. Discrete connection and covariant derivative for vector field analysis and design. ACM Trans. Graph. 35, 3 (Mar. 2016), 23:1-23:17.

X. Y. Liu, C. H. Lai, and K. A. Pericleous. 2015. A fourth-order partial differential equation denoising model with an adaptive relaxation method. Int. f. Comput. Math. 92, 3 (Mar. 2015), 608-622.

Charles Loop. 1987. Smooth Subdivision Surfaces Based on Triangles. Master's Thesis University of Utah.

M. Lysaker, A. Lundervold, and Xue-Cheng Tai. 2003. Noise removal using fourthorder partial differential equation with applications to medical magnetic resonance images in space and time. IEEE Trans. Image Proc. 12, 12 (Dec. 2003), 15791590.

Richard H. MacNeal. 1949. The Solution of Partial Differential Equations by Means of Electrical Networks. Ph.D. Dissertation. California Institute of Technology.

MATLAB. 2019. version 9.6 (R2019a). The MathWorks Inc.

Jorge Nocedal and Stephen J. Wright. 2006. Numerical Optimization (2nd ed.). Springer Science + Business Media, LLC

Pascal Peter, Sebastian Hoffmann, Frank Nedwed, Laurent Hoeltgen, and Joachim Weickert. 2016. Evaluating the true potential of diffusion-based inpainting in a compression context. Image Commun. 46, C (Aug. 2016), 40-53.

Peter Petersen. 2006. Riemannian Geometry (2nd ed.). Springer Science + Business Media, LLC.

Konrad Polthier and Markus Schmies. 1998. Straightest Geodesics on Polyhedral Surfaces. Springer Berlin, 135-150. DOI : https://doi.org/10.1007/978-3-662-03567-2 11

Alessio Quaglino. 2012. Membrane Locking in Discrete Shell Theories. Ph.D. Dissertation. Universität Göttingen.

Nicolas Ray, Bruno Vallet, Laurent Alonso, and Bruno Levy. 2009. Geometry-aware direction field processing. ACM Trans. Graph. 29, 1 (Dec. 2009), 1:1-1:11.

John William Strutt Rayleigh. 1894. The Theory of Sound. Vol. 1. Macmillan and Co.

Reinhard Scholz. 1978. A mixed method for 4th order problems using linear finite elements. RAIRO. Anal. numér. 12, 1 (1978), 85-90.

Nicholas Sharp, Yousuf Soliman, and Keenan Crane. 2018. The vector heat method. CoRR abs/1805.09170 (2018). Retrieved from http://arxiv.org/abs/1805.09170.

O. Sorkine, D. Cohen-Or, Y. Lipman, M. Alexa, C. Rössl, and H.-P. Seidel. 2004. Laplacian surface editing. In Proceedings of the Eurographics/ACM SIGGRAPH Symposium on Geometry Processing. ACM, New York, NY, 175-184.

Oded Stein, Eitan Grinspun, Max Wardetzky, and Alec Jacobson. 2018. Natural boundary conditions for smoothing in geometry processing. ACM Trans. Graph. 37, 2 (May 2018), 23:1-23:13

Daniel Sýkora, Ladislav Kavan, Martin Čadík, Ondřej Jamriška, Alec Jacobson, Brian Whited, Maryann Simmons, and Olga Sorkine-Hornung. 2014. Ink-and-ray: Basrelief meshes for adding global illumination effects to hand-drawn characters. ACM Trans. Graph. 33, 2 (Apr. 2014), Article 16, 15 pages. 
Amir Vaxman, Marcel Campen, Olga Diamanti, Daniele Panozzo, David Bommes, Klaus Hildebrandt, and Mirela Ben-Chen. 2016. Directional field synthesis, design, and processing. Comput. Graph. For. 35, 2 (2016), 545-572.

Yu Wang, Alec Jacobson, Jernej Barbič, and Ladislav Kavan. 2015. Linear subspace design for real-time shape deformation. ACM Trans. Graph. 34, 4 (2015), 57:157:11.

Yu Wang, Alec Jacobson, Jernej Barbič, and Ladislav Kavan. 2017. Error in "Linear Subspace Design for Real-time Shape Deformation.” Technical Report.

Max Wardetzky. 2006. Discrete Differential Operators on Polyhedral SurfacesConvergence and Approximation. Ph.D. Dissertation. FU Berlin.

Max Wardetzky, Saurabh Mathur, Felix Kälberer, and Eitan Grinspun. 2007. Discrete laplace operators: No free lunch. In Proceedings of the 5th Eurographics Symposium on Geometry Processing (SGP'07). Eurographics Association, 33-37.
Ofir Weber, Roi Poranne, and Craig Gotsman. 2012. Biharmonic coordinates. Comput. Graph. For. 31, 8 (2012), 2409-2422.

Ofir Weber, Olga Sorkine, Yaron Lipman, and Craig Gotsman. 2007. Context-aware skeletal shape deformationss. Comput. Graph. For. 26, 3 (2007), 265-274.

Tino Weinkauf, Yotam Gingold, and Olga Sorkine. 2010. Topology-based smoothing of 2D scalar fields with C1-continuity. Comput. Graph. For. 29, 3 (2010).

Eric W. Weisstein. 2019. Monge Patch. From MathWorld-A Wolfram Web Resource. Retrieved from http://mathworld.wolfram.com/MongePatch.html.

Roland Weitzenböck. 1885. Invariantentheorie. P. Noordhoff.

YahooJAPAN. 2013. Plane. Retrieved from https:/www.thingiverse.com/thing: 182252.

Received May 2019; revised January 2020; accepted January 2020 